\title{
Topological Quantum Fluctuations and Traveling Wave Amplifiers
}

\author{
Vittorio Peano, ${ }^{1,4}$ Martin Houde, ${ }^{2}$ Florian Marquardt, ${ }^{1,3}$ and Aashish A. Clerk ${ }^{2}$ \\ ${ }^{1}$ Institute for Theoretical Physics, University of Erlangen-Nürnberg, \\ Staudtstraße 7, 91058 Erlangen, Germany \\ ${ }^{2}$ Department of Physics, McGill University, 3600 rue University, Montreal, Quebec H3A 2T8, Canada \\ ${ }^{3}$ Max Planck Institute for the Science of Light, Günther-Scharowsky-Straße 1/Bau 24, \\ 91058 Erlangen, Germany \\ ${ }^{4}$ Department of Physics, University of Malta, Msida MSD 2080, Malta
}

(Received 29 April 2016; published 1 November 2016)

\begin{abstract}
It is now well established that photonic systems can exhibit topological energy bands. Similar to their electronic counterparts, this leads to the formation of chiral edge modes which can be used to transmit light in a manner that is protected against backscattering. While it is understood how classical signals can propagate under these conditions, it is an outstanding important question how the quantum vacuum fluctuations of the electromagnetic field get modified in the presence of a topological band structure. We address this challenge by exploring a setting where a nonzero topological invariant guarantees the presence of a parametrically unstable chiral edge mode in a system with boundaries, even though there are no bulkmode instabilities. We show that one can exploit this to realize a topologically protected, quantum-limited traveling wave parametric amplifier. The device is naturally protected against both internal losses and backscattering; the latter feature is in stark contrast to standard traveling wave amplifiers. This adds a new example to the list of potential quantum devices that profit from topological transport.
\end{abstract}

DOI: 10.1103/PhysRevX.6.041026

\section{INTRODUCTION}

The quantization of the electromagnetic field introduces a fundamentally new phenomenon into physics: vacuum fluctuations that permeate all of space. These fluctuations were initially seen as a basic unalterable feature of spacetime, before it was realized that they could be engineered to great effect. Simply modifying geometric boundary conditions changes the size of the fluctuations as a function of position and frequency, leading to phenomena such as the Purcell enhancement of spontaneous emission. The introduction of nonlinear optical materials gives rise to an even greater level of control, leading to the possibility of squeezed vacuum states [1], with important applications to sensing beyond the limits usually set by quantum mechanics [2-4].

In recent years, new approaches for altering the dynamics of wave fields have gained prominence, based on engineering periodic materials to elicit topological properties. Topologically protected unidirectional wave propagation was originally discovered in the study of 2D electrons in strong magnetic fields, and underlies the robust quantization of the Hall conductance [5]. The engineering of topological photonic materials has been the focus of intense theoretical investigation [6], and various experimental

Published by the American Physical Society under the terms of the Creative Commons Attribution 3.0 License. Further distribution of this work must maintain attribution to the author(s) and the published article's title, journal citation, and DOI.
Subject Areas: Condensed Matter Physics, Photonics, Topological Insulators platforms have already been developed [7-10]. Phononic topological states have also attracted recent attention [11-15], and the first experimental steps at the macroscopic scale have been taken [15-17].

Despite this considerable work in topological photonics and phononics, using topology to address the engineering of vacuum fluctuations has not been addressed. Most photonic and phononic topological systems are based on a singleparticle Hamiltonian that conserves particle number. These topological states mimic well-known electronic topological phases such as the quantum Hall phase [7,13,18-25] or the spin Hall phase [10,16,26-28] and have a trivial vacuum. In order to modify the properties of the vacuum, one has to introduce particle nonconserving terms to the Hamiltonian which can coherently add and remove pairs of particles from the system; these terms have a formal similarity to pairing terms in the mean-field description of a fermionic superconductor. If the amplitude of these terms is sufficiently weak, the system remains stable; even in this regime, the bosonic nature of the particles makes the topological properties of such Hamiltonians very different from their fermionic (topological superconductor) counterparts [29-32]. An even starker difference occurs when the parametric terms lead to dynamical instabilities [33-35]. These instabilities have no fermionic analogue and are akin to the parametric instability in an oscillator whose spring constant is modulated in time.

Here, we consider a situation where parametric driving is introduced to a system where photons hop on a lattice in the presence of a synthetic gauge field [see Fig. 1(a)]. We show how to realize an exotic situation where all bulk modes are 

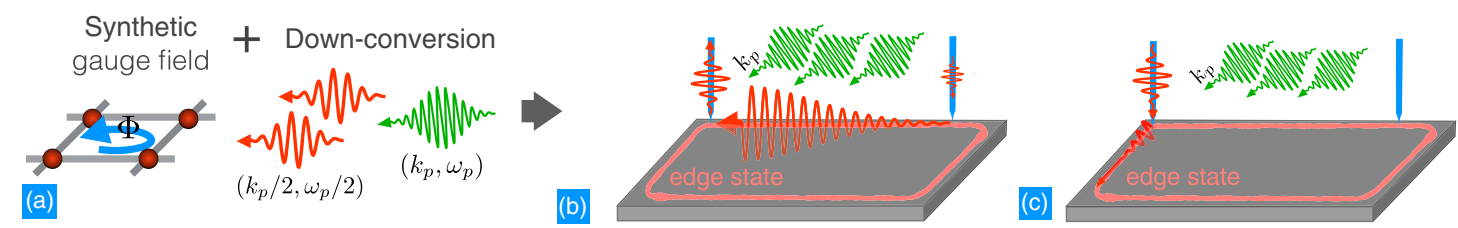

FIG. 1. Setup figure. (a) Scheme of the basic interactions. (i) Photons hopping anticlockwise around a plaquette pick up a phase $\Phi$ that can be interpreted as a synthetic gauge field flux. (ii) Pump photons with frequency $\omega_{p}$ and quasimomentum $k_{p}$ are down-converted into a photon pair with frequency $\omega_{p} / 2$ and quasimomentum $k_{p} / 2$. (b),(c) Combining the two interactions in a finite geometry allows one to engineer a topologically protected quantum-limited amplifier. A signal injected into the device via a tapered fiber propagates unidirectionally along the edge. (b) With the appropriate choice of pump frequency $\omega_{p}$ and quasimomentum $k_{p}$, the signal is amplified while it travels along the upper edge. A second tapered fiber detects the amplified signal. (c) When the input and the output fiber are exchanged, the signal propagates along a different path where it decays due to the lack of phase matching. This leads to nonreciprocal amplification.

stable, but where the topologically protected chiral edge modes that exist in a system with a boundary are unstable. This leads to an unusual spatially dependent modification of vacuum fluctuations: when the system is stabilized by dissipation, quantum fluctuations in the bulk are only weakly perturbed, whereas those along the system edge are strongly distorted. The result is not just an unusual driven-dissipative quantum state, but also a unique kind of photonic device: as we show in detail, the system serves both as a topologically protected, nonreciprocal, quantumlimited amplifier as well as a source of chiral squeezed light. It thus represents a potentially powerful new kind of application of topological materials.

\section{IDENTIFYING UNSTABLE MODES}

Before delving into the details of our proposal, it is useful to discuss the underlying theoretical ideas in a general setting. Our main goal is to exploit topological features of a dynamically unstable Hamiltonian, adding dissipation to realize a nonthermal steady state. In the absence of topological considerations, this is a situation that is ubiquitous in quantum optics. The simplest bosonic Hamiltonian exhibiting instability is the single-mode squeezing Hamiltonian:

$$
\hat{H}_{S}=\Delta \hat{a}^{\dagger} \hat{a}+\frac{i}{2} \nu\left(\hat{a}^{\dagger} \hat{a}^{\dagger}-\hat{a} \hat{a}\right)
$$

where $\hat{a}$ is a bosonic annihilation operator. Heuristically, $\hat{H}_{S}$ describes photons in a single cavity mode (effective energy $\Delta$ ) which are subject to coherent two-particle driving (with amplitude $\nu$ ). Without dissipation, $\hat{H}_{S}$ becomes unstable and cannot be diagonalized when the driving amplitude exceeds the energy cost for creating a pair of photons, i.e., when $\nu>|\Delta|$. In this regime, the dynamics corresponds to an ever-growing, exponential accumulation of entangled pairs of bosonic particles: there is no stationary state.

If we now add dissipation, stability can be restored by offsetting the effective two-particle driving described by $\hat{H}_{S}$ against the decay rate $\kappa$ of the mode; one requires $\kappa>2 \sqrt{\nu^{2}-\Delta^{2}}$. The result is a nonthermal stationary state having a steady flux of excitations flowing from the driven mode to the dissipative bath (which could be a waveguide serving as an input-output port). This is precisely the situation realized in a standard parametric amplifier: the linear-response properties of this driven-dissipative steady state allow for quantum-limited amplification of an additional signal drive. The required two-photon driving in Eq. (1) is generically realized by using a nonlinearity and parametric down-conversion of a driven pump mode.

\section{A. Normal mode decomposition of unstable Hamiltonians}

With these preliminaries, we now consider a very general quadratic Hamiltonian describing bosons on a lattice subject to parametric driving:

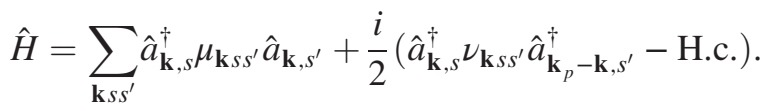

Here, the ladder operator $\hat{a}_{\mathbf{k}, s}$ annihilates a boson with quasimomentum $\mathbf{k}$ in the state $s$, where $s, s^{\prime}=1, \ldots, N$ label polarization and/or sublattice degrees of freedom. The first set of terms describes the hopping of photons on the lattice, and explicitly conserves both particle number and quasimomentum. It could be diagonalized to yield a standard band structure: for each quasimomentum $\mathbf{k}$, we have $N$ band eigenstates. The second set of parametric driving terms breaks particle number conservation, and, in general, also breaks the conservation of quasimomentum: the two-photon driving injects pairs with a net quasimomentum $\mathbf{k}_{p}$, implying that quasimomentum is conserved only modulo $\mathbf{k}_{p}$. For a realization based on a driven $\chi^{(2)}$ medium, the two-photon driving terms correspond to the down-conversion of pump photons with quasimomentum $\mathbf{k}_{p}$ into a pair of photons with quasimomenta $\mathbf{k}$ and $\mathbf{k}_{p}-\mathbf{k}$, respectively. Below, we show that having a 
nonzero quasimomentum for injected pairs is a crucial resource for controlling parametric instabilities.

Analogous to the simple Hamiltonian in Eq. (1), the lattice Hamiltonian in Eq. (2) exhibits instabilities when the amplitude for creating a pair of photons exceeds the energy of the pair. Formally, one can introduce a generalized normal mode decomposition of this generic Hamiltonian that explicitly separates out stable modes and unstable modes. One obtains (see Appendix A)

$$
\hat{H}=\sum_{\mathbf{k}} \sum_{n \in S_{\mathbf{k}}} E_{n, \mathbf{k}} \hat{n}_{n, \mathbf{k}}+\frac{1}{2} \sum_{n \in U_{\mathbf{k}}} \hat{H}_{n, \mathbf{k}} .
$$

For each quasimomentum $\mathbf{k}$ in the first Brillouin zone, we now have both a set of stable modes (indexed by $n \in S_{\mathbf{k}}$ ) and a set of unstable modes $\left(n \in U_{\mathbf{k}}\right)$. The first $n$ sum in Eq. (3) describes the stable modes: they are described by canonical bosonic anihiliation operators $\hat{\beta}_{n, \mathbf{k}}$ and enter the Hamiltonian in the standard manner, as a real energy times a number operator: $\hat{n}_{n, \mathbf{k}}=\hat{\beta}_{n, \mathbf{k}}^{\dagger} \hat{\beta}_{n, \mathbf{k}}$. The unstable modes can also be described by canonical bosonic anihiliation operators $\hat{\beta}_{n, \mathbf{k}}$. They, however, enter the Hamiltonian via unstable two-mode squeezing (parametric amplifier) Hamiltonians:

$$
\hat{H}_{n, \mathbf{k}}=E_{n, \mathbf{k}}\left(\hat{n}_{n, \mathbf{k}}-\hat{n}_{n, \mathbf{k}_{p}-\mathbf{k}}\right)+i \lambda_{n, \mathbf{k}}\left(\hat{\beta}_{n, \mathbf{k}}^{\dagger} \hat{\beta}_{n, \mathbf{k}_{p}-\mathbf{k}}^{\dagger}-\text { H.c. }\right),
$$

where $E_{n, \mathbf{k}}=-E_{n, \mathbf{k}_{p}-\mathbf{k}}$ and $\lambda_{n, \mathbf{k}}=\lambda_{n, \mathbf{k}_{p}-\mathbf{k}}$ are both real. This effective Hamiltonian for the unstable modes has a simple interpretation: pairs of quasiparticles with opposite energies $\pm E_{n, \mathbf{k}}$ are created with an amplitude $\lambda_{n, \mathbf{k}}$. We stress that for any nonzero $\lambda_{n, \mathbf{k}}, \hat{H}_{n, \mathbf{k}}$ is unstable (as the total energy for creating the relevant pair of excitations is always zero). The quasiparticle operators $\hat{\beta}_{n, \mathbf{k}}^{\dagger}$ in Eqs. (3) and (4) are a complete set of Bogoliubov ladder operators.

\section{B. Unstable Hamiltonians for cold atom systems}

A few pioneering works have discussed topological unstable Hamiltonians for cold atom systems in 1D $[33,35]$ and 2D [34]. In this framework, a Hamiltonian of the form of Eq. (2) (for $k_{p}=0$ ) emerges when the interactions between atoms in the condensed phase are treated at the mean-field level. In particular, Galilo et al. [34] discuss the interesting case of a 2D topological insulator whose only unstable modes are a pair of counterpropagating edge states. We emphasize that such unstable Hamiltonians do not describe steady-state behavior, but rather the transient exponential growth of population in the unstable modes after a quench. In contrast, the main focus of our work is on the nontrivial steady state that emerges when similar instabilities are stabilized by controlled dissipation.

\section{PARAMETRICALLY DRIVEN HOFSTADTER MODEL}

Having established the necessary theoretical framework, we now show how to engineer a Hamiltonian whose only unstable Bogoliubov modes $\hat{\beta}_{n, \mathbf{k}}^{\dagger}$ are chiral states copropagating along the physical boundary of a topological system. We consider photons hopping on a 2D square lattice in the presence of a synthetic magnetic field flux, which are also subject to parametric two-photon driving on each site [see Fig. 1(c)]. Writing the Hamiltonian in the position basis, we have

$\hat{H}=\sum_{\mathbf{j}} \omega_{\mathbf{j}} \hat{a}_{\mathbf{j}}^{\dagger} \hat{a}_{\mathbf{j}}-\sum_{\langle\mathbf{i}, \mathbf{j}\rangle} J_{\mathbf{i} \mathbf{j}} \hat{a}_{\mathbf{i}}^{\dagger} \hat{a}_{\mathbf{j}}+\frac{i}{2} \nu \sum_{\mathbf{j}}\left(e^{i \theta_{\mathbf{j}}} \hat{a}_{\mathbf{j}}^{\dagger} \hat{a}_{\mathbf{j}}^{\dagger}-\right.$ H.c. $)$,

where $\hat{a}_{\mathbf{j}}$ is the photon annihilation operator on site $\mathbf{j}=\left(j_{x}, j_{y}\right)$ and $\omega_{\mathbf{j}}$ are the corresponding on-site energies. As usual, the synthetic gauge field is encoded in the pattern of phases $\phi_{\mathbf{i j}}$ of the nearest-neighbor hopping rates, $J_{\mathbf{i j}}=J \exp \left(i \phi_{\mathrm{ij}}\right)$. We take the synthetic flux per plaquette to be $\Phi=\pi / 2$. Working in the Landau gauge, we then have $\phi_{\mathrm{ij}}=0$ for vertical hopping and $\phi_{\mathrm{ij}}=-\pi j_{y} / 2$ for rightwards hopping. The parametric driving amplitude on a given site $\mathbf{j}$ is written $\nu e^{i \theta_{\mathbf{j}}}$; we take the phase to vary as $\theta_{\mathbf{j}}=k_{p} j_{x}$, implying the injection of pairs with a quasimomentum $\mathbf{k}_{p}=k_{p} \mathbf{e}_{x}$. For a realization based on a driven nonlinear medium, $\nu \propto \sqrt{I_{p}}$, where $I_{p}$ is the power of the pump laser, and $k_{p} \mathbf{e}_{x}$ is the quasimomentum of the pump laser photons. Note that a gauge transformation $\hat{a}_{\mathbf{j}}^{\prime}=\hat{a}_{\mathbf{j}} \exp \left[i f_{\mathbf{j}}\right]$ would modify both phase fields $\phi_{\mathrm{ij}}$ and $\theta_{\mathbf{j}}$.

When the laser is switched off, $\nu=0$, and there is no disorder, $\omega_{\mathbf{j}}=\omega_{0}$, we have the well-known Hofstadter model [36]. The band structure of a semi-infinite strip (extending to the lower-half 2D plane) is shown in Fig. 2(a). The continuous bulk band structure consists of four bands (one for each site in the magnetic unit cell). The top and bottom bands are flat Landau levels separated from the two central bands by topological band gaps. Because of the boundary, one finds inside each topological band gap an edge state (dark line). The net number of these edge states (the number weighted by the sign of their slope) is a topologically protected quantity that does not depend on the shape of the edge and can be calculated from the bulk Hamiltonian [37].

We now turn on the parametric driving such that the resulting Hamiltonian can exhibit instability. Our goal is twofold: we want the system to be unstable only if we have a boundary, and in this case, the dominant unstable Bogoliubov modes should be chiral excitations localized at the system's boundary. We do this by choosing the parametric drive parameters so that the only pairs of photons that can be created in an energy and 


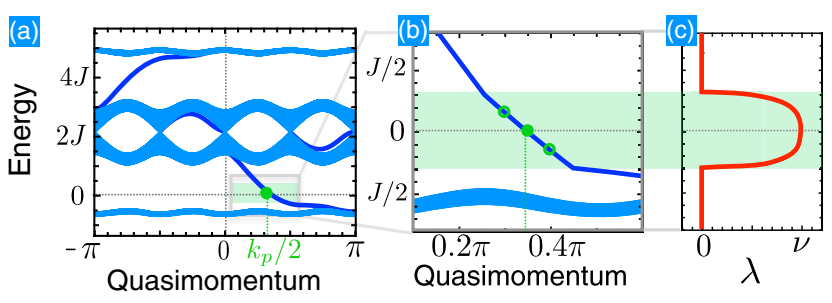

FIG. 2. Topological band structure. (a) Band structure of a semi-infinite strip for the Hofstadter model with a flux of $\pi / 2$ (when the parametric driving is switched off). The edge states are plotted in dark blue. The energy is counted off from half of the pump photon energy. The solid green circle indicates the tuning of the pump photon quasimomentum $k_{p}$ required to resonantly excite pairs of down-converted edge state photons with quasimomentum $k_{p} / 2$ (at the quasimomentum $k_{p} / 2$ the edge state should have energy $E=0$ ). (b) Zoom of the band structure for a finite laser power. In the unstable energy interval highlighted in green, pairs of Bogoliubov excitations having quasimomenta $k_{p} / 2 \pm \delta k$ are also excited. The corresponding amplification amplitude $\lambda$ is shown in (c). Parameters are $\omega_{0}=2.15 \mathrm{~J}$, $\Phi=\pi / 2, k_{p}=2.2$. In (b) and (c), $\nu=0.08 \mathrm{~J}$.

quasimomentum conserving fashion correspond to edge state excitations of the original $(\nu=0)$ model. For concreteness, we focus on exciting the edge mode in the lower topological band gap [dispersion $\varepsilon_{E}(k)$ ]. In the lab frame, we thus tune the pump photon frequency $\omega_{p}$ and quasimomentum $k_{p}$ so that a single pump photon can be converted into two edge excitations with frequency $\omega_{p} / 2$ and quasimomentum $k_{p} / 2$. In the rotating frame we use to write our Hamiltonians, this requirement reduces to $\varepsilon_{E}\left(k_{p} / 2\right)=0$. If this resonance condition is met, an arbitrarily weak parametric drive $\nu$ will cause instability of the edge mode. The required tuning is shown in Fig. 2(a).

Because of the approximately linear dispersion relation of the edge mode, the above tuning guarantees that the parametric driving can resonantly create a pair of edge mode photons having momenta $k_{p} / 2 \pm \delta k$; see the hollow circles in Fig. 2(b). Thus, even for a weak parametric drive amplitude, the edge state will exhibit instability over a range of quasimomenta near $k_{p} / 2$ (corresponding to a finite bandwidth around $\omega_{p} / 2$ in the lab frame); see Fig. 2(c).

Conversely, the energies of two bulk excitations always add up to a finite value; see Appendix B. In other words, all bulk parametric transitions have a finite detuning. This guarantees the bulk stability (even in the presence of disorder) for a driving amplitude $\nu$ below the minimal value of the bulk detuning.

\section{TOPOLOGICAL NONRECIPROCAL AMPLIFIER}

Having shown how to realize an unstable topological edge mode, we now want to understand how one can use it. More precisely, we show that a finite-size array of
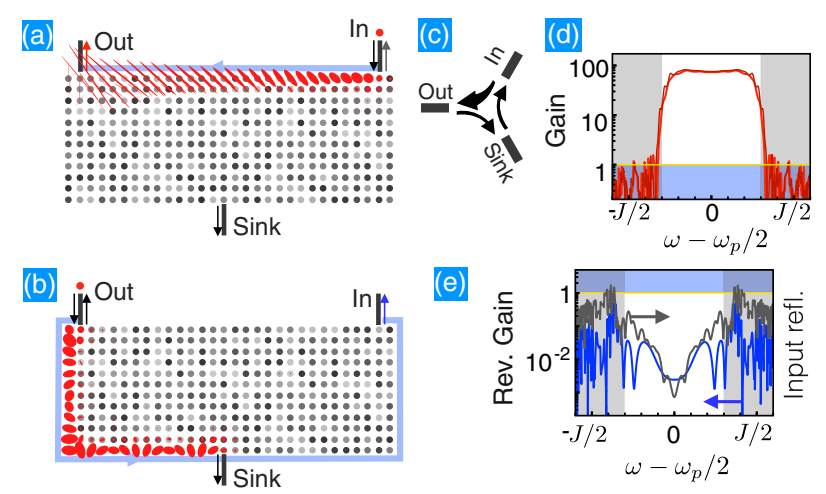

FIG. 3. Linear response of the topological amplifier. (a),(b) The topological amplifier is formed by a $30 \times 12$ array of photonic nanocavities. Three of the cavities at the edge of the sample are attached to waveguides, the input and output ports of the amplifier, and an additional sink port. (c) The amplifier has the geometry of a circulator. (a),(b) The red ellipses represent the linear response of the field inside the photonic array as a function of the incoming signal phase. The signal is injected at the port marked by an inward arrow. (a) A signal injected at the input port propagates unidirectionally toward the output port. The response is strongly phase sensitive; a signal with the right phase is amplified along the way. (d) Transmission power gain for the amplified quadrature as a function of the frequency of the input signal (counted off from half of the pump frequency) for a disordered (light thick line) and a clean sample (dark thin line). The reflection coefficient at the input is shown in (e) for the disordered sample. (b) A signal propagating from the output port toward the input port follows a different path and it is not amplified. Moreover, an appropriate matching of the impedances ensures that it leaks out at the sink port. The resulting (small) reverse transmission from the output to the input of the amplifier is the blue curve in (e). Parameters are $\omega_{0}=2.14 J, \Phi=\pi / 2$, $\nu=0.08 \mathrm{~J}, \quad k_{p}=2.2, \quad \kappa=0.001 \mathrm{~J}, \quad \kappa_{\text {in }}=2.6 \mathrm{~J}, \quad \kappa_{\text {out }}=3 \mathrm{~J}$, $\kappa_{\text {sink }}=4.2 \mathrm{~J}$. In the disordered simulations, the offset energies $\delta \omega_{\mathbf{j}}$, represented by the gray scale in (a) and (b), are random numbers in the interval $-0.1 J<\delta \omega_{\mathbf{j}}<0.1 J$.

nanocavities coupled to simple waveguides can be used as a new kind of topologically protected, phase-sensitive, quantum-limited amplifier. The role of the waveguides is twofold: they serve as amplifier input-output ports and they stabilize the dynamics.

We consider a realization of our system using a $30 \times 12$ array of nanocavities, and additionally include three coupling waveguides. Each waveguide is coupled to a site at the edge of the sample, as shown in Figs. 3(a) and 3(b). This coupling is described using standard input-output theory, and is entirely characterized by the three rates $\kappa_{\text {in }}$, $\kappa_{\text {out }}$, and $\kappa_{\text {sink }}$; see Appendix C. In addition, we take each cavity to have an internal-loss decay rate $\kappa$.

When the small decay rate $\kappa$ is neglected, and without parametric driving, the array can be operated as an ideal circulator where a signal from any waveguide is entirely transmitted into the next waveguide; see Fig. 3(c). Indeed, it is always possible to match the impedances at each port to 
cancel the backreflection by tuning the corresponding coupling rate $\left(\kappa_{\mathrm{in}}, \kappa_{\text {out }}\right.$, or $\left.\kappa_{\text {sink }}\right)$. Once inside the array, a wave in a topological band gap has no alternative but to chirally propagate along the edge. In addition, the impedance matching ensures that a wave impinging on a waveguide from the edge channel will be entirely transmitted.

We harness the robust nonreciprocity of this topological circulator to design an amplifier. We use the waveguide on the upper right (left) as an input (output) port of the amplifier. We choose parametric driving parameters similar to Fig. 2. In the finite geometry, the quasimomentum matching will be approximately realized only on the upper edge. Thus, the amplification occurs mainly in the region between the input and the output port.

The linear response of the amplifier is investigated numerically in Fig. 3. A signal injected into the array from the input port propagates chirally until it leaves the array through the output port; see Fig. 3(a). Depending on its phase, it can be amplified or deamplified along the way. Treating the amplifier as a phase-sensitive amplifier, we find that the power gain for the amplified signal quadrature is flat over a large bandwidth, corresponding to the frequency range over which the edge state dispersion is purely linear [see Fig. 3(d)]. At the same time, any signals incident upon the output port will be almost entirely dumped into the sink port, and not reach the input port; see Fig. 3(b). The residual reverse gain and input reflection are much smaller than unity, see Fig. 3(e), ensuring the protection of a potentially fragile signal source (e.g., a qubit). Crucially, this strongly nonreciprocal amplifying behavior is of topological origin and is thus robust against disorder. We demonstrate this resilience by including moderate levels of disorder in our simulations (see Fig. 3).

Our numerical results are in qualitative agreement with analytical results for a model in which a 1D chiral edge state is coupled to three waveguides; see Appendix D. In this context, we find simple expressions for the impedancematching condition and the maximum power gain,

$$
\kappa_{i}=\frac{4 v}{\left|u\left(j_{y}=-1\right)\right|^{2}}, \quad G \approx \exp \left[\frac{2 \nu L}{v}\right],
$$

respectively. Here, $u\left(j_{y}\right)$ is the transverse edge state wave function, $v$ is the edge state velocity, and $L$ is the number of sites separating the input and the output ports. Thus, we see that the gain is the exponential of the rate $2 \nu$ of creation of down-converted pairs times the time of flight $L / v$ from the input to the output port. While we focus here on operation as a phase-sensitive amplifier, for frequencies different from $\omega_{p} / 2$, one could also use the device as a quantumlimited phase-preserving (i.e., nondegenerate) amplifier.

\section{A. Quantum-limited amplification}

The noise floor of our amplifier is determined by the spectral density of the amplified quadrature of the field leaving the output waveguide; see Appendix $\mathrm{C}$ for more details. It is plotted in dark (light) red for a clean (disordered) sample in Fig. 4(a). The quantum limit on a phase-sensitive amplifier is to have zero added noise [38], implying that the noise floor is simply set by the amplification of the vacuum fluctuations entering the input port. The added noise (expressed as an equivalent number of input quanta) is plotted in Fig. 4(b); despite disorder and noise associated with internal loss, the amplifier is nearly quantum limited over the entire amplification bandwidth.
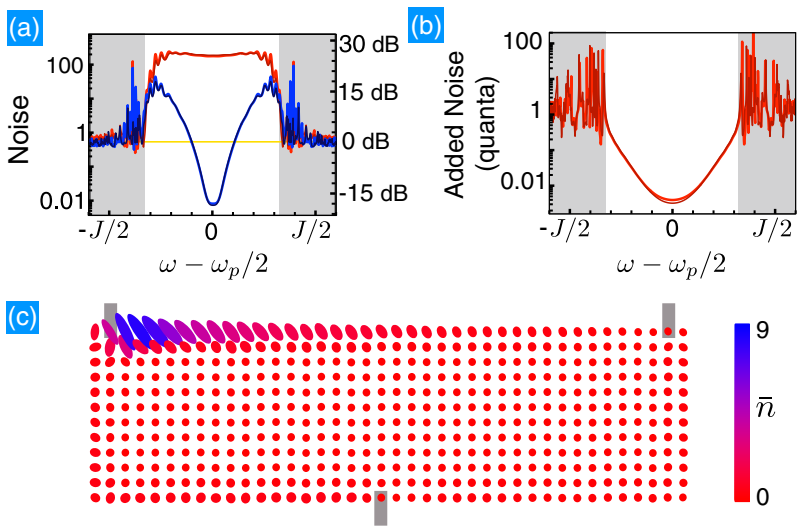

FIG. 4. Quantum stationary state and noise properties of the topological amplifier. (a) Noise spectral densities for both the amplified (red) and squeezed (blue) quadratures of the field leaving the output waveguide as a function of frequency, cf. Eq. (C9); the noise is plotted in units of quanta (left-hand axis), and also in decibels relative to the vacuum noise level (right-hand axis). The field leaking out of the output waveguide [top left corner in (c)] is strongly squeezed. We plot results for both a disordered system (light thick lines) and a clean sample (dark thin lines). The noise in the amplified quadrature is only slightly larger than the quantum-limit value for a phasepreserving amplifier (i.e., the amplified vacuum noise from the input port). The added noise in the amplified quadrature [in units of quanta; cf. Eq. (C10))] for both a disordered and a clean sample is shown in (b). (c) Ellipses representing the Gaussian Wigner function of state of each site inside the cavity array [cf. Eq. (C11)]. In the bulk, the ellipses have a circular shape and their area is as small as allowed by the Heisenberg principle, representing a standard vacuum state. In contrast, the ellipses at the edge are anisotropic and have areas larger than the minimum required by the uncertainty principle, implying that one has a thermal squeezed state. This excess noise does not come from a finite temperature of the environment, but rather by the amplification of the zero-point fluctuations (quantum heating). Plotted as a color code is also the average number of photons on each site. The gray bars indicate the sites that are attached to coupling waveguides. Parameters are $40 \times 12$ sites, $\omega_{0}=2.14 J, \Phi=\pi / 2, \quad \nu=0.08 J, \quad k_{p}=2.2$, $\kappa=0.001 J, \kappa_{\text {in }}=2.6 J, \kappa_{\text {out }}=3 J, \kappa_{\text {sink }}=4.2 J$. For the disordered simulations the offset energies $\delta \omega_{\mathbf{j}}$ are random numbers in the interval $-0.1 J<\delta \omega_{\mathbf{j}}<0.1 J$. For all plots, there is only vacuum noise entering from each waveguide. 


\section{TOPOLOGICAL STATIONARY STATE}

Next, we investigate the quantum fluctuations in the stationary state that arise from the steady flow of photons from the parametric pump to the amplifier ports in the form of down-converted radiation. Physically, such a flow arises because vacuum fluctuations entering the input port (within the amplification bandwidth) are amplified inside the array before exiting through the output port. The resulting stationary state of each cavity is Gaussian, and can be represented by a Wigner function; these are visualized as a set of noise ellipses in Fig. 4(c). The nonzero eccentricity of these ellipses indicates that the noise is maximally large in one direction in phase space (corresponding to the amplified quadrature), and a minimum in the orthogonal direction (the deamplified, or "squeezed" quadrature). Each noise ellipse completely characterizes the steady state of the corresponding site once all remaining sites and the waveguides have been traced out. The areas of the ellipses are constrained from below by the Heisenberg uncertainty principle and assume the minimal possible value for pure states. The bulk sites are all in the trivial quantum ground state, which is characterized by circular ellipses with the minimal area and zero photons. On the other hand, the eccentricity, the area, and the average on-site photon number (color code) grow while moving from the input to the output port along the upper edge (the major axis corresponds to the amplified quadrature). We emphasize that, in a thermal equilibrium setting, the area of the ellipses would be equal on all sites and directly reflect the environment temperature. Here, the excess noise of the sites on the upper edge has a quantum origin. This phenomenon has been termed quantum heating [39-41].

Because of quantum heating, the stationary state of each cavity along the edge corresponds to a thermal squeezed state, implying that the deamplified, or squeezed, quadrature has a larger variance than the minimum required by the uncertainty principle. Nonetheless, the frequencyresolved output noise is strongly squeezed below the vacuum level (for frequencies within the amplification bandwidth). Remarkably, the quality of the output squeezing is not deteriorated in the presence of disorder. For the parameters we consider here, more than $15 \mathrm{~dB}$ of output squeezing are predicted in both the case of a clean and a disordered sample; see the blue curves in Fig. 4(a).

\section{IMPLEMENTATION}

Photonic gauge fields have already been realized in several experimental platforms $[7,9,10]$. The only additional ingredient of our proposal is the parametric pumping with a finite quasimomentum. In the setup of Ref. [10], one could fabricate the microrings from a nonlinear optical $\chi^{(2)}$ material and drive them with a single laser impinging at a finite angle. The implementation of parametric pumping [32] and synthetic gauge fields $[13,20,22,25,32]$ is in principle possible in any cavity array platform where a nonlinear resource is available. These include photonic crystal microcavities [42] fabricated from nonlinear optical $\chi^{(2)}$ materials [43-45] or piezoelectric materials, and optomechanical arrays based on optomechanical crystals [46,47]. An alternate route would be to use lattices of superconducting resonators with embedded Josephson nonlinearities [48]. To that end, we note the recent work by Anderson et al. [49], which outlines a detailed experimental proposal for constructing a square lattice of lowloss 3D cavities with exactly the value of synthetic flux required by our proposal. Crucially, the synthetic flux is obtained without any time-dependent driving. By adding Josephson junctions to this setup (as is also proposed in Ref. [49]), one has an extremely promising venue for realizing the topological amplifier physics discussed here. We also note that very recently a proposal for realizing topological insulator physics in a classical optical network with nonlinearities was put forward [50]; such a setup could also be adapted to implement our scheme, as it contains all the necessary ingredients.

\section{CONCLUSIONS AND OUTLOOK}

In this work, we introduce a means to tie the squeezing and amplification of vacuum fluctuations to topological properties of a band structure. Our work represents a new design principle for a nonreciprocal quantum-limited amplifier that has topological protection. Nonreciprocal amplifiers have the potential to revolutionize experiments with superconducting qubits, as they could eliminate the need for ferrite-based circulators and the accompanying insertion losses that limit current experiments. A variety of (nontopological) designs based on multiple parametric interactions have been proposed recently [51,52], and even realized experimentally $[53,54]$, including a traveling wave parametric amplifier built using an array of over 2000 Josephson junctions [55]. In such a conventional traveling wave parametric amplifier, the reverse transmission is at best unity, and even small amounts of disorder can lead to large amounts of unwanted reflection gain. In contrast, the topological underpinnings of our design ensure reverse transmission and input reflection coefficients that are well below unity even in the presence of disorder. Our design also has advantages over cavity-based approaches, namely, the lack of any fundamental constraint on its gain-bandwidth product.

More generally, our topological amplifier differs markedly from other proposed topological devices, such as isolators or nonamplifying circulators [6], in that it has some protection against internal losses: in the large gain limit, only the loss (and corresponding noise) in the immediate vicinity of the input port hinders quantumlimited operations, as it is only this noise that is amplified to any significant degree (see Appendix D for a quantitative discussion of this point). 
In conclusion, our work shows how utilizing the topological properties of an unstable bosonic Hamiltonian provides a new route for both engineering electromagnetic vacuum fluctuations and, correspondingly, constructing a new kind of topologically protected, nonreciprocal quantum amplifier. It opens the door to future studies, pursuing both other kinds of novel applications as well as more fundamental issues (e.g., the effects of additional photonphoton interactions in such systems).

\section{ACKNOWLEDGMENTS}

V. P. and F. M. acknowledge support by an ERC Starting Grant OPTOMECH, by the DARPA project ORCHID, and by the European Marie-Curie ITN network cQOM. M. H. and A. A. C. acknowledge support from NSERC. We thank Ignacio Cirac, Sebastian Huber, and André Xuereb for discussion.

\section{APPENDIX A: GENERALIZED NORMAL MODE DECOMPOSITION}

We consider the generic Hamiltonian Eq. (2). We group all ladder operators with quasimomentum $\mathbf{k}$ in a vector of ladder operators, $\left|\hat{a}_{\mathbf{k}}\right\rangle=$ $\left(\hat{a}_{\mathbf{k}, 1}, \ldots, \hat{a}_{\mathbf{k}, N}, \hat{a}_{\mathbf{k}_{p}-\mathbf{k}, 1}^{\dagger}, \ldots, \hat{a}_{\mathbf{k}_{p}-\mathbf{k}, N}^{\dagger}\right)^{T}$. The Heisenberg equation of motion for $\left|\hat{a}_{\mathbf{k}}\right\rangle$ reads

$$
\frac{d}{d t}\left|\hat{a}_{\mathbf{k}}\right\rangle=-i \sigma_{\mathrm{z}} h_{\mathbf{k}}\left|\hat{a}_{\mathbf{k}}\right\rangle
$$

where

$$
\sigma_{\mathrm{z}}=\left(\begin{array}{cc}
\mathbb{1}_{N} & 0 \\
0 & -\mathbb{1}_{N}
\end{array}\right)
$$

and $h_{\mathbf{k}}$ is the Bogoliubov-de Gennes (BdG) Hamiltonian

$$
h_{\mathbf{k}}=\left(\begin{array}{cc}
\mu_{\mathbf{k}} & i \nu_{\mathbf{k}} \\
-i \nu_{\mathbf{k}}^{\dagger} & \mu_{\mathbf{k}_{p}-\mathbf{k}}
\end{array}\right) .
$$

In the following, we explicitly construct a complete set of Bogoliubov operators $\hat{\beta}_{n, \mathbf{k}}$, leading to the generalized normal mode decomposition Eq. (3), from the solutions of the eigenvalue problem:

$$
\sigma_{\mathrm{z}} h_{\mathbf{k}}\left|\mathbf{k}_{n, l}\right\rangle=\Lambda_{\mathbf{k}, n, l}\left|\mathbf{k}_{n, l}\right\rangle .
$$

We note that we need to find only $N$ annihilation operators while the eigenvalue problem has dimension $2 N$. However, the equations for quasimomentum $\mathbf{k}_{p}-\mathbf{k}$ are not independent from the equations for quasimomentum $\mathbf{k}$. One set of these equations can be obtained from the other by taking the adjoint. This doubling of the degrees of freedom accompanied by an embedded particle-hole symmetry occurs because we are effectively applying a single-particle formalism to a problem where the number of excitations is not conserved.

From Eq. (A2) it is easy to prove that eigenvalues that are not related by complex conjugation, $\Lambda_{n, l, \mathbf{k}} \neq \Lambda_{n^{\prime}, l^{\prime}, \mathbf{k}}^{*}$, have $\sigma_{z}$ orthogonal eigenvectors, $\left\langle\mathbf{k}_{n, l}\left|\sigma_{z}\right| \mathbf{k}_{n^{\prime}, l^{\prime}}\right\rangle=0$. Moreover, the eigenvectors with real eigenvalues have a nonzero symplectic norm, $\left\langle\mathbf{k}_{n, l}\left|\sigma_{\mathrm{z}}\right| \mathbf{k}_{n, l}\right\rangle \neq 0$ (which can also be negative). We assign the label $l=+$ to the positive norm eigenvectors. We construct a set of orthonormal Bogoliubov creation operators from these positive norm solutions with the definition

$$
\hat{\beta}_{n, \mathbf{k}} \equiv\left\langle\mathbf{k}_{n,+}\left|\sigma_{z}\right| \hat{a}_{\mathbf{k}}\right\rangle .
$$

We note that the scalar product between a standard vector and a vector of operators is an operator. Moreover, we have to normalize the positive vectors $\left|\mathbf{k}_{n, l}\right\rangle$ according to $\left\langle\mathbf{k}_{n,+}\left|\sigma_{\mathrm{z}}\right| \mathbf{k}_{n,+}\right\rangle=1$ such that $\left[\hat{\beta}_{n, \mathbf{k}}, \hat{\beta}_{n^{\prime}, \mathbf{k}}^{\dagger}\right]=\delta_{n n^{\prime}}$. By taking the time derivative of Eq. (A3) and plugging Eq. (A1) and the adjoint of Eq. (A2), we immediately find

$$
\dot{\hat{\beta}}_{n, \mathbf{k}}=-i\left\langle\mathbf{k}_{n,+}\left|\sigma_{z} \sigma_{z} h_{\mathbf{k}}\right| \hat{a}_{\mathbf{k}}\right\rangle=-i \Lambda_{n,+, \mathbf{k}} \hat{\beta}_{n, \mathbf{k}} .
$$

Thus, $\hat{\beta}_{n, \mathbf{k}}$ is the annihilation operator of a harmonic oscillator with energy $E_{n, \mathbf{k}}=\Lambda_{n,+, \mathbf{k}}$. In the same way, one could construct a set of creation operators $\hat{\beta}_{n, \mathbf{k}_{p}-\mathbf{k}}^{\dagger}$ from the negative norm eigenvectors $\left|\mathbf{k}_{n,-}\right\rangle$. However, it is possible to focus only on the positive norm solutions because of the particle-hole symmetry: the information encoded in the negative norm solutions $\left|\mathbf{k}_{n,-}\right\rangle$ is also encoded in the positive norm solutions $\left|\left(\mathbf{k}_{p}-\mathbf{k}\right)_{n,+}\right\rangle$.

The remaining eigenvectors have zero norm, $\left\langle\mathbf{k}_{n, \pm}\left|\sigma_{\mathrm{z}}\right| \mathbf{k}_{n, \pm}\right\rangle=0$. They appear whenever the Hamiltonian is unstable. In this case, the matrix $\sigma_{z} h_{\mathbf{k}}$ has pairs of complex conjugated eigenvalues, $\Lambda_{n,+, \mathbf{k}}=\Lambda_{n,-, \mathbf{k}}^{*}$. For concreteness, we indicate with the label + the eigenvalues with positive imaginary part. The pair of eigenvectors $\left|\mathbf{k}_{n, \pm}\right\rangle$ are not orthogonal to each other, $\left\langle\mathbf{k}_{n,+}\left|\sigma_{\mathrm{z}}\right| \mathbf{k}_{n,-}\right\rangle \neq 0$. In this case, we define a pair of commuting ladder operators as

$$
\begin{array}{r}
\hat{\beta}_{n, \mathbf{k}} \equiv \frac{1}{\sqrt{2}}\left(\left\langle\mathbf{k}_{n,-}\left|+i\left\langle\mathbf{k}_{n,+}\right|\right) \sigma_{z} \mid \hat{a}_{\mathbf{k}}\right\rangle,\right. \\
\hat{\beta}_{n, \mathbf{k}_{p}-\mathbf{k}}^{\dagger} \equiv \frac{1}{\sqrt{2}}\left(\left\langle\mathbf{k}_{n,-}\left|-i\left\langle\mathbf{k}_{n,+}\right|\right) \sigma_{z} \mid \hat{a}_{\mathbf{k}}\right\rangle .\right.
\end{array}
$$

The bosonic commutation relations are recovered by requiring the normalization $\left\langle\mathbf{k}_{n,-}\left|\sigma_{\mathrm{z}}\right| \mathbf{k}_{n,+}\right\rangle=i$. By taking the time derivative of Eq. (A4) and using Eq. (A1), the transpose of Eq. (A2), and Eq. (A5), we find

$$
\dot{\hat{\beta}}_{n, \mathbf{k}}=-i \operatorname{Re}\left[\Lambda_{n,+, \mathbf{k}}\right] \hat{\beta}_{n, \mathbf{k}}+\operatorname{Im}\left[\Lambda_{n,+, \mathbf{k}}\right] \hat{\beta}_{n, \mathbf{k}_{p}-\mathbf{k}}^{\dagger} .
$$


Likewise, we find

$$
\dot{\hat{\beta}}_{n, \mathbf{k}_{p}-k}^{\dagger}=-i \operatorname{Re}\left[\Lambda_{n,+, \mathbf{k}}\right] \hat{\beta}_{n, \mathbf{k}_{p}-k}^{\dagger}+\operatorname{Im}\left[\Lambda_{n,+, \mathbf{k}}\right] \hat{\beta}_{n, \mathbf{k}_{p}-\mathbf{k}} .
$$

The corresponding Hamiltonian is the two-mode squeezing Hamiltonian [Eq. (4)], with energies $E_{n, \mathbf{k}}=-E_{n, \mathbf{k}_{p}-\mathbf{k}}=$ $\operatorname{Re}\left[\Lambda_{n,+, \mathbf{k}}\right]$ and amplification amplitude $\lambda_{n, \mathbf{k}}=\operatorname{Im}\left[\Lambda_{n,+, \mathbf{k}}\right]$.

When the matrix $\sigma_{z} h$ is diagonalizable, the set of Bogoliubov annihilation operators defined in Eqs. (A3) and (A4) is complete. The pathological case where the matrix $\sigma_{z} h$ is not diagonalizable occurs only exactly at the threshold of an instability.

\section{APPENDIX B: DETAILS OF THE CALCULATION OF THE BAND STRUCTURE}

\section{Stability of the bulk Hamiltonian}

In the main text, we explain that a bosonic Hamiltonian with anomalous pairing terms is unstable when it allows the creation of a pair of Bogoliubov excitations without any net energy change. In other words, the sum of two quasiparticle energies should be zero. We also claim that for the parameters of Fig. 2 the bulk Hamiltonian is stable because no combination of bulk states whose energies add up to zero exists. This is not immediately obvious from the plot of the standard band structure. In order to visually illustrate the absence of such a combination of bulk states, one has to, rather, plot the corresponding Bogoliubov-de Gennes band structure; see Fig. 5. For each value of $k_{x}$, the latter is formed by both the quasiparticle energies $E_{n, k_{x}}$ (blue bands) as well as the energies $-E_{n, k_{p}-k_{x}}$ (gray bands). In analogy to the descriptions of quasiparticles in fermionic superconductors, one refers to the former (latter) as the particle (hole) bands of the system. The absence of crossings between particle and hole bands for the chosen pump laser frequency implies that there is no combination of a pair of bulk quasiparticles whose energies add up to zero while at the same time the corresponding quasimomenta add up to $k_{p}$. In the limit of vanishing parametric driving $(\nu=0)$, the minimal distance between particle and hole bands is the minimal detuning of a parametric transition involving two bulk states.

In the presence of weak disorder, there is no selection rule for the quasimomenta of the pair of quasiparticles created in a parametric transition. Nevertheless, all possible parametric transitions are still detuned because the band gap separating particle and hole bands is not merely locally defined (for a fixed $k_{x}$ ) but rather extends to the whole Brillouin zone. Thus, the stability of the bulk Hamiltonian is a robust feature.

As we explain in Appendix A, the Bogoliubov-de Gennes band structure can be calculated by diagonalizing the matrix $\sigma_{z} h\left(k_{x}\right)$, where $h\left(k_{x}\right)$ is the first-quantized Bogoliubov-de Gennes Hamiltonian equivalent to the second-quantized Hamiltonian Eq. (5) of the main text in the presence of periodic boundary conditions both in the $x$ and $y$ directions.

\section{Band structure of a strip}

Figure 2 represents the band structure and the amplification amplitudes of a semi-infinite strip. Those are derived from the Bogoliubov-de Gennes band structure and the amplification rates of a strip with two physical edges, as we explain below.

We simulate a finite strip of width $M=40$ magnetic unit cells. For each value of the quasimomentum $k_{x}$, the set of energies $E_{n, k_{x}}$ and $-E_{n, k_{p}-k_{x}}$ forming the BdG band structure and the corresponding amplification rates $\lambda_{n}\left(k_{x}\right)$ are calculated by diagonalizing the relevant $4 M \times 4 M$ matrix $\sigma_{z} h\left(k_{x}\right)$. While, strictly speaking, the energy spectrum is discrete, the spacing between subsequent bulk states is not visible on the figure scale. The resulting band structure and the corresponding amplification rates are shown in Figs. 5(b)5(c) and Fig. 5(e), respectively. By inspecting the corresponding wave functions, one can easily distinguish between particle and hole bands (plotted in blue and gray,
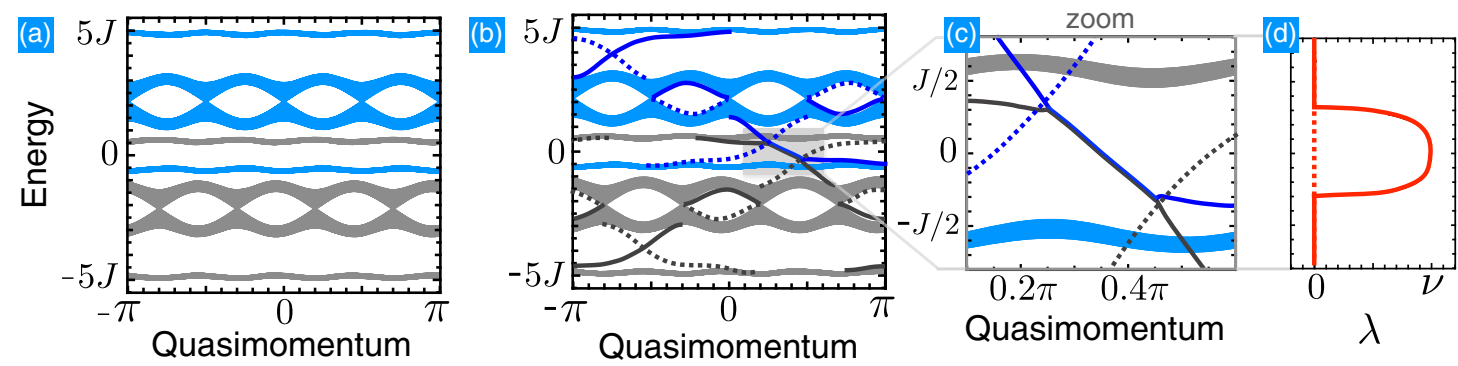

FIG. 5. Bogoliubov-de Gennes band structures. (a) BdG band structure for a system without boundaries. Here and in (b) and (c), the particle (hole) bands are plotted in blue (gray). The stability of the bulk is protected by the complete band gaps separating neighboring particle and hole bands. (b),(c) BdG band structure of a finite strip of width $M=40$ magnetic unit cells. The upper- (lower-)edge states are represented as thick solid (dashed) lines. (d) Corresponding amplification amplitudes $\lambda_{n}$. The parameters are the same as for Fig. 2 $\left(\omega_{0}=2.15 J, \Phi=\pi / 2, k_{p}=2.2, \nu=0.08 J\right)$. 
respectively), edge and bulk states (plotted by the thick dark and thin light lines, respectively), and upper- and lower-edge states (solid and dashed lines, respectively).

Since the hole bands are not independent from the particle bands and refer to the same set of normal modes, we do not display the hole energies in the plots in the main text. Moreover, we show only the edge states localized on the upper edge. Removing the edge states localized on the lower edge effectively corresponds to plotting the band structure of a semi-infinite strip extending to the lowerhalf plane.

We note that the particle (hole) edge states cross the hole (particle) band. Thus, a pair formed by an edge and a bulk excitation can in principle also be resonantly excited. However, the corresponding matrix element is very small and the resulting amplification rate is not visible on the scale of the figure.

\section{Analytical derivation of edge state dispersion and amplification rate}

Here, we outline a direct derivation of the edge state dispersion for a semi-infinite strip.

We first consider the case where the parametric driving is switched off $(\nu=0)$. This first part of the derivation follows the approach of Ref. [56]. The Hamiltonian in terms of the annihilation operators $\hat{a}_{j_{y}, k_{x}}$ of a plane wave with quasimomentum $k_{x}$ on the $\left|j_{y}\right|$ th row reads

$$
\begin{aligned}
\hat{H}= & \sum_{j_{y}, k_{x}}\left[\omega_{0}-2 J \cos \left(k_{x}+\pi j_{y} / 2\right)\right] \hat{a}_{j_{y}, k_{x}}^{\dagger} \hat{a}_{j_{y}, k_{x}} \\
& -J\left(\hat{a}_{j_{y}-1, k_{x}}^{\dagger} \hat{a}_{j_{y}, k_{x}}+\hat{a}_{j_{y}, k}^{\dagger} \hat{a}_{j_{y}-1, k_{x}}\right) .
\end{aligned}
$$

Since the strip extends to the lower-half plane, the $j_{y}$ th sum runs over the negative integers. By definition of the normal modes $\hat{\alpha}_{n, k_{x}}$ and eigenenergies $E_{n}\left[k_{x}\right]$, we have $\hat{H}=\sum_{n, k_{x}} E_{n}\left[k_{x}\right] \hat{\alpha}_{n, k_{x}}^{\dagger} \hat{\alpha}_{n, k_{x}}$, or, equivalently, $\left[\hat{\alpha}_{n, k_{x}}, \hat{H}\right]=$ $E_{n}\left[k_{x}\right] \hat{\alpha}_{n, k_{x}}$. By plugging the ansatz $\hat{\alpha}_{n, k_{x}}^{\dagger}=$ $\sum_{j_{y}} u_{n, k_{x}}\left[j_{y}\right] \hat{a}_{j_{y}, k_{x}}^{\dagger}$, we arrive to the first-quantized timeindependent Schrödinger equation, which we set in the matrix form:

$$
\begin{aligned}
\left(\begin{array}{c}
u_{n, k_{x}}\left[j_{y}-1\right] \\
u_{n, k_{x}}\left[j_{y}\right]
\end{array}\right)= & \tilde{M}_{j_{y}}\left(\epsilon_{n}\left[k_{x}\right]\right)\left(\begin{array}{c}
u_{n, k_{x}}\left[j_{y}\right] \\
u_{n, k_{x}}\left[j_{y}+1\right]
\end{array}\right) \\
= & \left(\begin{array}{cc}
-\epsilon_{n}\left[k_{x}\right]-2 \cos \left(k_{x}+\pi j_{y} / 2\right) & -1 \\
1 & 0
\end{array}\right) \\
& \times\left(\begin{array}{c}
u_{n, k_{x}}\left[j_{y}\right] \\
u_{n, k_{x}}\left[j_{y}+1\right]
\end{array}\right) .
\end{aligned}
$$

Here, we define $\epsilon_{n}\left[k_{x}\right]=\left(E_{n}\left[k_{x}\right]-\omega_{0}\right) / J$. In principle, the above equation holds for $j_{y} \leq-2$ but not for $j_{y}=-1$, because there is no row corresponding to $j_{y}=0$. One can circumvent this problem by formally adding the row $j_{y}=0$ together with the boundary condition $u_{n, k_{x}}[0]=0$. Thus, one immediately finds

$$
\left(\begin{array}{c}
u_{n, k_{x}}\left[j_{y}\right] \\
u_{n, k_{x}}\left[j_{y}-1\right]
\end{array}\right)=\prod_{\left|j_{y}^{\prime}\right|<\left|j_{y}\right|} \tilde{M}_{j_{y}^{\prime}}\left(\epsilon_{n}\left[k_{x}\right]\right)\left(\begin{array}{c}
u_{n, k_{x}}[-1] \\
0
\end{array}\right) .
$$

Next, we focus on the edge state solutions. In the following, we drop the subscript $n$ because there is only one edge state in each band gap; see below. For the edge states, we have to enforce the boundary condition

$$
\lim _{j_{y} \rightarrow-\infty} u_{k_{x}}\left[j_{y}\right]=0 .
$$

Equivalently, one can require

$$
\begin{aligned}
& \lim _{m \rightarrow \infty}\left(\begin{array}{c}
u_{k_{x}}[-4 m-1] \\
u_{k_{x}}[-4 m]
\end{array}\right) \\
& =\lim _{m \rightarrow \infty}\left[M\left(\epsilon\left[k_{x}\right]\right)\right]^{m}\left(\begin{array}{c}
u_{k_{x}}[-1] \\
0
\end{array}\right)=0,
\end{aligned}
$$

where $M\left(\epsilon\left[k_{x}\right]\right)$ is the transfer matrix by a full magnetic unit cell (four sites),

$M\left(\epsilon\left[k_{x}\right]\right)=\tilde{M}_{-4}\left(\epsilon\left[k_{x}\right]\right) \tilde{M}_{-3}\left(\epsilon\left[k_{x}\right]\right) \tilde{M}_{-2}\left(\epsilon\left[k_{x}\right]\right) \tilde{M}_{-1}\left(\epsilon\left[k_{x}\right]\right)$.

Equation (B5) is fulfilled if and only if the vector $(1,0)$ is an eigenvector of the 4-site transfer matrix $M\left(\epsilon\left[k_{x}\right]\right)$ whose eigenvalue has modulus smaller than unity. In other words, the edge state dispersion is determined by the conditions

$$
\begin{aligned}
M_{2,1}\left(\epsilon\left[k_{x}\right]\right)= & -\epsilon\left(-4+\epsilon^{2}+2 \cos \left[2 k_{x}\right]\right) \\
& +2 \cos \left[k_{x}\right]\left(\epsilon^{2}-4 \sin \left[k_{x}\right]^{2}\right)=0, \\
\left|M_{1,1}\left(\epsilon\left[k_{x}\right]\right)\right|= & \mid \epsilon^{4}-7 \epsilon^{2}-2 \epsilon\left(\cos \left[k_{x}\right]+\sin \left[k_{x}\right]\right) \\
& +3+2 \sin \left[2 k_{x}\right]-4 \cos \left[4 k_{x}\right] \mid<1 .
\end{aligned}
$$

Equation (B7) is a third-order polynomial in the dimensionless energy $\epsilon$. Thus, it has three roots for each value of $k_{x}$. Each root corresponds to a solution inside one of the three band gaps (lower, middle, or upper). By plugging the analytical expression of each root of Eq. (B7) into Eq. (B8), we find the range of $k_{x}$ where the corresponding edge state is defined: the interval $-\pi<k_{x}<0$ for the upper edge state, $0<k_{x}<\pi$ for the lower edge state, and the two intervals $-\pi / 2<k_{x}<0$ and $\pi / 2<k_{x}<\pi$ for the edge state in the local band gap between the two central bands. In order to enforce the bosonic commutation relations $\left[\hat{\alpha}_{k_{x}}, \hat{\alpha}_{k_{x}}^{\dagger}\right]=1$, we have to appropriately normalize the single-particle wave functions $\sum_{j_{y}}\left|u_{k_{x}}\left[j_{y}\right]\right|^{2}=1$. This 
condition fixes the modulus of the wave function on the initial site $u_{k_{x}}[-1]$. The complex phase of $u_{k_{x}}[-1]$ is arbitrary. For concreteness, we choose $u_{k_{x}}[-1]$ to be real.

Next, we show how the edge state is modified by the parametric pump. The pump drive written in terms of the annihilation operators $\hat{a}_{j_{y}, k_{x}}$ reads

$$
\hat{H}_{\text {pump }}=i \frac{\nu}{2} \sum_{j_{y}, k_{x}}\left(\hat{a}_{j_{y}, k_{x}}^{\dagger} \hat{a}_{j_{y}, k_{p}-k_{x}}^{\dagger}-\hat{a}_{j_{y}, k_{x}} \hat{a}_{j_{y}, k_{p}-k_{x}}\right) .
$$

Before pursuing an exact numerical solution, we adopt a semianalytical treatment. We focus on the lower band gap edge state, which is resonantly driven for the parameters of Fig. 2. As in the main text, we consider the case where the frequency and quasimomentum of the pump laser are chosen to resonantly excite a pair of edge photons with quasimomentum $k_{p} / 2$ :

$$
\omega_{0}=-J \varepsilon\left[k_{p} / 2\right] .
$$

In this case, it is convenient to introduce the quasimomentum $\delta k=k_{x}-k_{p} / 2$ counted off from $k_{p} / 2$. We rewrite the full Hamiltonian (including the pump) in terms of the ladder operators of the eigenstates of the Hofstadter Hamiltonian (without the pump). We focus on the edge state's ladder operators $\hat{\alpha}_{\delta k}$ and neglect pump interactions, which include bulk modes and the other edge modes (the dominating interaction is that which resonantly excites a pair of edge state photons). Doing so, we find, $H^{(\text {edge })}=\sum_{\delta k} H_{\delta k}^{(\text {edge })} / 2$,

$$
\begin{aligned}
H_{\delta k}^{(\text {edge })}= & \tilde{E}_{n}(\delta k)\left(\hat{\alpha}_{\delta k}^{\dagger} \hat{\alpha}_{\delta k}-\hat{\alpha}_{-\delta k}^{\dagger} \hat{\alpha}_{-\delta k}\right) \\
& +\Delta[\delta k]\left(\hat{\alpha}_{\delta k}^{\dagger} \hat{\alpha}_{\delta k}+\hat{\alpha}_{-\delta k}^{\dagger} \hat{\alpha}_{-\delta k}\right) \\
& +i V[\delta k]\left(\hat{\alpha}_{\delta k}^{\dagger} \hat{\alpha}_{-\delta k}^{\dagger}-\hat{\alpha}_{\delta k} \hat{\alpha}_{-\delta k}\right) .
\end{aligned}
$$

Here, we expand the edge state energy Eq. (B10) around $k_{p} / 2$ and group all excitation conserving terms into two contributions whose amplitudes $\tilde{E}_{n}(\delta k)$ and $\Delta_{n}(\delta k)$ are an odd and even function of $\delta k$, respectively:

$$
\begin{aligned}
\tilde{E}_{n}(\delta k) & =\left.J \sum_{n \geq 1} \frac{d^{2 n-1} \varepsilon}{d k_{x}^{2 n-1}}\right|_{k_{p} / 2} \frac{\delta k^{2 n-1}}{(2 n-1) !}, \\
\Delta(\delta k) & =\left.J \sum_{n \geq 1} \frac{d^{2 n} \varepsilon}{d k_{x}^{2 n}}\right|_{k_{p} / 2} \frac{\delta k^{2 n}}{(2 n) !} .
\end{aligned}
$$

Note that $\Delta(\delta k)$ is the quasi-momentum-dependent detuning of the parametric transition creating pairs of photons with quasimomentum $k_{p} / 2 \pm \delta k$. The corresponding parametric coupling, expressed in terms of the edge state wave function, is given by

$$
V(\delta k)=\nu \sum_{j_{y}} u_{\delta k}\left[j_{y}\right] u_{-\delta k}\left[j_{y}\right]
$$

Next, we expand the above formula in powers of $\delta k$. The leading term is $\nu$ because the wave functions $u_{\delta k}$ are normalized. Moreover, $V$ is obviously an even function of $\delta k$ (it is invariant if $\delta k$ is replaced by $-\delta k$ ). Thus, the expansion has the form

$$
V(\delta k)=\nu-\sum_{n \geq 1} \nu_{n} \delta k^{2 n} .
$$

The edge state is unstable over the finite quasimomentum interval where $V(\delta k)>|\Delta(\delta)|$. Following the general procedure presented in Appendix A, we find the eigenenergies and amplification amplitude of the Bogoliubov edge state normal modes

$$
\begin{aligned}
& E(\delta k)=\tilde{E}(\delta k), \\
& \lambda(\delta k)=\sqrt{[V(\delta k)]^{2}-[\Delta(\delta k)]^{2},}
\end{aligned}
$$

and

$$
E(\delta k)=\tilde{E}(\delta k)+\sqrt{[\Delta(\delta k)]^{2}-[V(\delta k)]^{2}},
$$

in the unstable and stable quasimomentum ranges, respectively.

We can recover the edge state dispersion and line shape of the amplification amplitude obtained numerically and shown in Fig 2 by keeping the leading-order contributions in Eqs. (B12), (B13), and (B15):

$$
\begin{aligned}
& V(\delta k) \approx \nu-\nu_{1} \delta k^{2}, \quad \nu_{1}=0.014, \\
& \tilde{E}(\delta k) \approx v \delta k, \quad \frac{v}{J}=\left.\frac{d \varepsilon}{d k_{x}}\right|_{k_{p} / 2}=-1, \\
& \left.\Delta(\delta k) \approx J \frac{d^{2} \varepsilon}{d k_{x}^{2}}\right|_{k_{p} / 2} \frac{\delta k^{2}}{2},\left.\quad \frac{d^{2} \varepsilon}{d k_{x}^{2}}\right|_{k_{p} / 2}=1.4 .
\end{aligned}
$$

\section{APPENDIX C: INPUT-OUTPUT FORMALISM}

We include the effects of dissipation using the standard input-output formalism [1]. Each site is described by the Langevin equation:

$$
\dot{\hat{a}}_{\mathbf{j}}=i\left[\hat{H}, \hat{a}_{\mathbf{j}}\right]-\kappa_{\mathbf{j}} \hat{a}_{\mathbf{j}} / 2+\sqrt{\kappa_{\mathbf{j}}} \hat{a}_{\mathbf{j}}^{(\text {in) }} .
$$

Here, $\kappa_{\mathbf{j}}$ is the decay rate on site $\mathbf{j}$. On the sites coupled to waveguides, the decay rates $\kappa_{\text {in }}, \kappa_{\text {out }}$, and $\kappa_{\text {sink }}$ are induced by the coupling to the waveguides and are chosen to achieve impedance matching. In this case, the input fields $\hat{a}_{\mathbf{j}}^{(\text {in) }}$ describe the field impinging on site $\mathbf{j}$ from the 
corresponding waveguide including the field vacuum fluctuations. The field $\hat{a}_{\mathbf{j}}^{\text {(out) }}$ leaking out of the waveguide is given by the input-output relations

$$
\hat{a}_{\mathbf{j}}^{(\text {out })}=\hat{a}_{\mathbf{j}}^{(\text {in })}-\sqrt{\kappa_{\mathbf{j}}} \hat{a}_{\mathbf{j}} .
$$

On the remaining sites (i.e., those not coupled to waveguides), we assume a small uniform decay rate $\kappa\left(\kappa_{\mathbf{j}}=\kappa\right)$ corresponding to internal loss; $\hat{a}_{\mathbf{j}}^{(\mathrm{in})}$ describes the corresponding incident vacuum fluctuations associated with this loss port.

The linear response and the noise properties of the array are simple functions of the retarded Green's functions:

$$
\begin{aligned}
& \tilde{G}_{E}\left(\omega, \mathbf{j}, \mathbf{j}^{\prime}\right)=-i \int_{-\infty}^{\infty} d t \Theta(t) e^{i \omega t}\left\langle\left[\hat{a}_{\mathbf{j}}(t), \hat{a}_{\mathbf{j}^{\prime}}^{\dagger}(0)\right]\right\rangle, \\
& \tilde{G}_{I}\left(\omega, \mathbf{j}, \mathbf{j}^{\prime}\right)=-i \int_{-\infty}^{\infty} d t \Theta(t) e^{i \omega t}\left\langle\left[\hat{a}_{\mathbf{j}}^{\dagger}(t), \hat{a}_{\mathbf{j}^{\prime}}^{\dagger}(0)\right]\right\rangle .
\end{aligned}
$$

We calculate the above Green's functions numerically for a finite array.

\section{Response ellipses}

In Fig. 3(a) [Fig. 3(b)], we show the linear response of the pair of quadratures,

$$
\begin{aligned}
& \hat{X}_{\mathbf{j}}=\frac{1}{\sqrt{2}}\left[e^{i \theta_{\mathbf{j}} / 2} \hat{a}_{\mathbf{j}}^{\dagger}(t)+e^{-i \theta_{\mathbf{j}} / 2} \hat{a}_{\mathbf{j}}(t)\right], \\
& \hat{Y}_{\mathbf{j}}=\frac{i}{\sqrt{2}}\left[e^{i \theta_{\mathbf{j}} / 2} \hat{a}_{\mathbf{j}}^{\dagger}(t)-e^{-i \theta_{\mathbf{j}} / 2} \hat{a}_{\mathbf{j}}(t)\right]
\end{aligned}
$$

to a classical field of frequency $\omega_{p} / 2$ injected at the input (output) port as a function of the field phase $\vartheta$,

$$
\left\langle\hat{a}_{\mathbf{j}^{\prime}}^{(\mathrm{in})}\right\rangle=\sqrt{\kappa_{\mathbf{j}^{\prime}}} e^{i \vartheta},
$$

where $\mathbf{j}^{\prime}$ indicates the site attached to the relevant injection waveguide $\left[\mathbf{j}^{\prime}=\mathbf{j}_{\text {in }}\right.$ for Fig. 3(a), $\mathbf{j}^{\prime}=\mathbf{j}_{\text {out }}$ for Fig. 3(b)]. The response on each site is represented by the ellipse

$$
\left(\begin{array}{c}
\left\langle\hat{X}_{\mathbf{j}}\right\rangle \\
\left\langle\hat{Y}_{\mathbf{j}}\right\rangle
\end{array}\right)=\left(\begin{array}{cc}
\cos \gamma_{\mathbf{j} \mathbf{j}^{\prime}} & -\sin \gamma_{\mathbf{j} \mathbf{j}^{\prime}} \\
\sin \gamma_{\mathbf{j} \mathbf{j}^{\prime}} & \cos \gamma_{\mathbf{j} \mathbf{j}^{\prime}}
\end{array}\right)\left(\begin{array}{cc}
r_{\mathbf{j} \mathbf{j}^{\prime}}^{+} & \cos \left(\vartheta-\eta_{\mathbf{j} \mathbf{j}^{\prime}}\right) \\
r_{\mathbf{j} \mathbf{j}^{\prime}}^{-} & \sin \left(\vartheta-\eta_{\mathbf{j j}^{\prime}}\right)
\end{array}\right),
$$

where

$$
r_{\mathbf{j j}^{\prime}}^{ \pm}=\kappa_{\mathbf{j}^{\prime}}\left(\left|\tilde{G}_{E}\left(0, \mathbf{j}, \mathbf{j}^{\prime}\right)\right| \pm\left|\tilde{G}_{I}\left(0, \mathbf{j}, \mathbf{j}^{\prime}\right)\right|\right)
$$

are the major and minor semiaxes of the ellipse, and

$$
\gamma_{\mathbf{j} \mathbf{j}^{\prime}}=\arg \left(\frac{G_{E}\left(0, \mathbf{j}, \mathbf{j}^{\prime}\right)}{G_{I}\left(0, \mathbf{j}, \mathbf{j}^{\prime}\right)}\right) / 2-\theta_{\mathbf{j}} / 2
$$

is the angle parametrizing the orientation of the major semiaxis. In the figure, the reference angle $\gamma_{\mathbf{j j}^{\prime}}=0$ is rotated by $\pi / 2$ compared to the page vertical. The maximum (minimum) response $r_{\mathbf{j} \mathbf{j}^{\prime}}^{+}\left(r_{\mathbf{j} \mathbf{j}^{\prime}}^{-}\right)$at site $\mathbf{j}$ to an input field at the port $\mathbf{j}^{\prime}$ is obtained for the driving phase $\vartheta=\eta_{\mathbf{j} \mathbf{j}^{\prime}}$ $\left(\vartheta=\eta_{\mathbf{j} \mathbf{j}^{\prime}}+\pi / 2\right)$ :

$$
\eta_{\mathbf{j} \mathbf{j}^{\prime}}=\pi / 2-\arg \left[\left(G_{E}\left(0, \mathbf{j}, \mathbf{j}^{\prime}\right) G_{I}\left(0, \mathbf{j}, \mathbf{j}^{\prime}\right)\right] / 2 .\right.
$$

\section{Gain, reverse gain, and input reflection}

As we analyze our amplifier in the phase-sensitive mode of operation, it is useful to consider its scattering properties in a quadrature representation, using optimal quadrature basis for the fields in the input and output waveguides. The relevant scattering between these waveguides is described by

$$
\begin{aligned}
& \hat{\mathcal{X}}_{\text {out }}[\omega]=s_{\mathcal{X}, \mathcal{U}}[\omega] \hat{\mathcal{U}}_{\text {in }}[\omega]+s_{\mathcal{X}, \mathcal{V}}[\omega] \hat{\mathcal{V}}_{\text {in }}[\omega]+\cdots, \\
& \hat{\mathcal{Y}}_{\text {out }}[\omega]=s_{\mathcal{Y}, \mathcal{U}}[\omega] \hat{\mathcal{U}}_{\text {in }}[\omega]+s_{\mathcal{Y}, \mathcal{V}}[\omega] \hat{\mathcal{V}}_{\text {in }}[\omega]+\cdots,
\end{aligned}
$$

where we omit writing terms describing contributions from vacuum noise incident from the internal loss ports, as well as terms describing the reflection of noise incident from the output waveguide (due to slightly imperfect impedance matching). We define the quadratures of the output waveguide as

$$
\begin{aligned}
& \hat{\mathcal{X}}_{\text {out }}(t)=\frac{1}{\sqrt{2}}\left[e^{i \theta_{\text {out }}} \hat{a}_{\mathbf{j}_{\text {out }}}^{\text {(out }) \dagger}(t)+e^{-i \theta_{\text {out }}} \hat{a}_{\mathbf{j}_{\text {out }}}^{\text {out })}(t)\right], \\
& \hat{\mathcal{Y}}_{\text {out }}(t)=\frac{i}{\sqrt{2}}\left[e^{i \theta_{\text {out }}} \hat{a}_{\mathbf{j}_{\text {out }}}^{\text {out }) \dagger}(t)-e^{-i \theta_{\text {out }}} \hat{a}_{\mathbf{j}_{\text {out }}}^{(\text {out })}(t)\right],
\end{aligned}
$$

while the quadratures of the input waveguide are defined as

$$
\begin{aligned}
& \hat{\mathcal{U}}_{\mathrm{in}}(t)=\frac{1}{\sqrt{2}}\left[e^{i \theta_{\mathrm{in}}} \hat{a}_{\mathbf{j}_{\mathrm{in}}}^{(\mathrm{in}) \dagger}(t)+e^{-i \theta_{\mathrm{in}}} \hat{a}_{\mathbf{j}_{\mathrm{in}}}^{(\mathrm{in})}(t)\right], \\
& \hat{\mathcal{V}}_{\mathrm{in}}(t)=\frac{i}{\sqrt{2}}\left[e^{i \theta_{\mathrm{in}}} \hat{a}_{\mathbf{j}_{\mathrm{in}}}^{(\mathrm{in}) \dagger}(t)-e^{-i \theta_{\mathrm{in}}} \hat{a}_{\mathbf{j}_{\mathrm{in}}}^{(\mathrm{in})}(t)\right] .
\end{aligned}
$$

We define both the quadrature basis in each coupling waveguide so that $\left|s_{\mathcal{X}, \mathcal{U}}[\omega=0]\right|$ is maximal. This implies that the largest amplification of a narrow-band signal centered at zero frequency ( $\omega_{p} / 2$ in the lab frame) occurs when the incident signal is in the $\hat{\mathcal{U}}_{\text {in }}$ quadrature of the input waveguide, with the amplified output being contained in the output field $\hat{\mathcal{X}}_{\text {out }}$ quadrature of the output waveguide. It also follows naturally that for vacuum noise inputs, $\hat{\mathcal{Y}}_{\text {out }}$ will be the optimally squeezed quadrature. An explicit calculation of the scattering matrix in terms of the Green's functions introduced above shows that the angles defining these preferred input and output quadratures are given by

$$
\theta_{\text {in }}=\eta_{\mathbf{j}_{\text {out }} \mathbf{j}_{\text {in }}}, \quad \theta_{\text {out }}=\gamma_{\mathbf{j}_{\text {out }} \mathbf{j}_{\text {in }}}+\theta_{\mathbf{j}_{\text {out }}} / 2
$$


see Eqs. (C6) and (C7). The fact that, in general, $\theta_{\text {in }} \neq \theta_{\text {out }}$ is a simple reflection of the fact that our system can both perform phase-sensitive amplification of incident signals as well as simply rotate incident signals in phase space.

The frequency-dependent power gain is then by definition given by the transmission probability:

$$
\begin{aligned}
G(\omega)= & \left|s_{\mathcal{X}, \mathcal{U}}(\omega)\right|^{2} \\
= & \kappa_{\text {in }} \kappa_{\text {out }} \mid e^{i\left(\theta_{\text {in }}-\theta_{\text {out }}\right)} G_{E}\left(\omega, \mathbf{j}^{(\text {out })}, \mathbf{j}^{\text {(in) })}\right. \\
& +e^{i\left(\theta_{\text {in }}+\theta_{\text {out }}\right)} G_{I}\left(\omega, \mathbf{j}^{\text {(out) }}, \mathbf{j}^{\text {(in) }}\right) \\
& +e^{i\left(\theta_{\text {out }}-\theta_{\text {in }}\right)} G_{E}^{*}\left(-\omega, \mathbf{j}^{(\text {out })}, \mathbf{j}^{(\text {in })}\right) \\
& +e^{-i\left(\theta_{\text {in }}+\theta_{\text {out }}\right)} G_{I}^{*}\left(-\omega, \mathbf{j}^{\text {(out) }},\left.\mathbf{j}^{\text {(in) })}\right|^{2} / 4 .\right.
\end{aligned}
$$

The reverse gain is obtained similarly by exchanging the two indices of the Green's functions in the above formula and in Eq. (C8). Finally, the input reflection probability describes the reflection of signals incident in the (amplified) $\mathcal{U}$ quadrature of the input port

$$
\begin{aligned}
R[\omega]= & \left|s_{\mathcal{U}, \mathcal{U}}(\omega)\right|^{2}=\left\{\mid e^{i \theta_{\text {in }}}\left[1-i \kappa_{\text {in }} G_{E}\left(\omega, \mathbf{j}^{(\text {out })}, \mathbf{j}^{(\text {in })}\right)\right]\right. \\
& +e^{-i \theta_{\text {in }}}\left[1+i \kappa_{\text {in }} G_{I}^{*}\left(-\omega, \mathbf{j}^{\text {(out) }}, \mathbf{j}^{\text {(in) })}\right)\right] \mid \\
& +\mid e^{i \theta_{\text {in }}}\left[1-i \kappa_{\text {in }} G_{I}\left(\omega, \mathbf{j}^{\text {(out) })}, \mathbf{j}^{(\text {in })}\right)\right] \\
& \left.+e^{-i \theta_{\text {in }}}\left[1+i \kappa_{\text {in }} G_{E}^{*}\left(-\omega, \mathbf{j}^{(\text {out })}, \mathbf{j}^{(\text {in })}\right)\right] \mid\right\}^{2} / 4 .
\end{aligned}
$$

\section{Noise spectral densities and amplifier added noise}

In general, the output of an amplifier will consist of the amplified input signal (including its intrinsic fluctuations) plus extra fluctuations generated by the amplifier [38]. It is standard to express these extra output fluctuations as an effective number of noise quanta inserted at the amplifier input: this is the "added noise quanta of the amplifier." For a phase-sensitive amplifier like ours (which only amplifies one quadrature of an input signal), quantum mechanics allows the added noise to be arbitrarily small. Approaching this limit of zero added noise thus represents the "amplifier quantum limit" for this kind of amplifier. See, e.g., Ref. [57] for an extended pedagogical discussion of these topics.

The symmetrized noise spectral densities for the amplified and deamplified output-waveguide output field quadratures [as shown in Fig. 4(a)] are defined as

$$
\begin{aligned}
& S_{\mathcal{X}, \mathcal{X}}(\omega)=\int_{-\infty}^{\infty} \frac{d t}{2} e^{i \omega t}\left\langle\left\{\hat{\mathcal{X}}_{\text {out }}(t), \hat{\mathcal{X}}_{\text {out }}(0)\right\}\right\rangle, \\
& S_{\mathcal{Y}, \mathcal{Y}}(\omega)=\int_{-\infty}^{\infty} \frac{d t}{2} e^{i \omega t}\left\langle\left\{\hat{\mathcal{Y}}_{\text {out }}(t), \hat{\mathcal{Y}}_{\text {out }}(0)\right\}\right\rangle .
\end{aligned}
$$

To obtain the amplifier added noise at a given frequency [as shown in Fig. 4(b)], we take the output noise in the amplified quadrature at this frequency, divide through by the gain at this frequency (so as to have an effective amount of noise at the input of the amplifier), and then subtract off the contribution due to vacuum noise entering through the input port (as this is part of the input signal, as opposed to noise added by the amplifier). We thus obtain

$$
S_{\text {add }}(\omega) \equiv \frac{S_{\mathcal{X}, \mathcal{X}}(\omega)}{G(\omega)}-\frac{1}{2} .
$$

This quantity approaches zero for a quantum-limited phase preserving amplifier.

\section{Noise ellipses}

In Fig. 4(c), the stationary state of each site $\mathbf{j}$ is represented as a noise ellipse. The noise ellipse on a particular site $\mathbf{j}$ is obtained by diagonalizing the corresponding covariance matrix,

$$
\begin{aligned}
V_{\mathbf{j}}= & \left(\begin{array}{cc}
\left\langle\hat{X}_{\mathbf{j}}^{2}\right\rangle & \left\langle\hat{X}_{\mathbf{j}} \hat{Y}_{\mathbf{j}}+\hat{Y}_{\mathbf{j}} \hat{X}_{\mathbf{j}}\right\rangle \\
\left\langle\hat{X}_{\mathbf{j}} \hat{Y}_{\mathbf{j}}+\hat{Y}_{\mathbf{j}} \hat{X}_{\mathbf{j}}\right\rangle & \left\langle\hat{Y}_{\mathbf{j}}^{2}\right\rangle
\end{array}\right) \\
= & \left(\begin{array}{cc}
\cos \tilde{\gamma}_{\mathbf{j}} & \sin \tilde{\gamma}_{\mathbf{j}} \\
-\sin \tilde{\gamma}_{\mathbf{j}} & \cos \tilde{\gamma}_{\mathbf{j}}
\end{array}\right)\left(\begin{array}{cc}
\left(\tilde{r}_{\mathbf{j}}^{+}\right)^{2} & 0 \\
0 & \left(\tilde{r}_{\mathbf{j}}^{-}\right)^{2}
\end{array}\right) \\
& \times\left(\begin{array}{cc}
\cos \tilde{\gamma}_{\mathbf{j}} & -\sin \tilde{\gamma}_{\mathbf{j}} \\
\sin \tilde{\gamma}_{\mathbf{j}} & \cos \tilde{\gamma}_{\mathbf{j}}
\end{array}\right),
\end{aligned}
$$

and identifying the square root of its eigenvalues $\tilde{r}_{\mathbf{j}}^{+}$and $\tilde{r}_{\mathbf{j}}^{-}$ as the ellipse semiaxes and the angle $\tilde{\gamma}_{\mathbf{j}}$ as the ellipse rotation angle (the reference angle $\tilde{\gamma}_{\mathbf{j}}=0$ is rotated by $\pi / 2$ compared to the page vertical).

\section{APPENDIX D: EFFECTIVE MODEL FOR THE EDGE STATE COUPLED TO WAVEGUIDES}

Here, we derive an effective quantum field theory for the edge state coupled to a waveguide. We want to model a finite system that will then support a single edge state with a ring geometry (i.e., periodic boundary conditions). We adopt the simplest possible approach valid when the input signal has a bandwidth well within the amplifier bandwidth. In this case, we can approximate the edge state velocity and amplification rates to be constant, $\tilde{E} \approx v \delta k, \Delta \approx 0$, and $V(\delta k) \approx \nu$, respectively. Moreover, we can neglect the quasimomentum dependence of the edge state transverse wave function. We thus arrive at the edge state ladder operator,

$\hat{c}\left(j_{\|}\right)=\sqrt{\frac{1}{N}} \sum_{n=1}^{N} e^{i \delta k_{n} j_{\|}} \hat{\alpha}_{\delta k} \approx e^{-i \kappa_{p} j_{\|} / 2} \sum_{j_{\perp}} u\left[j_{\perp}\right] \hat{a}_{j_{\|}, j_{\perp}}$,

where $N$ is the number of sites along the edge and the indices $j_{\|}$and $j_{\perp}\left(j_{\perp} \geq 1\right)$ label the position in the directions parallel and longitudinal to the edges (for the edge state along the upper edge described above, $j_{\|}=j_{x}$ and 
$\left.j_{\perp}=-j_{y}\right)$. Moreover, $u\left(j_{\perp}\right)$ is the transverse edge state wave function, $u\left(j_{\perp}\right) \equiv u_{k_{p} / 2}\left(-j_{\perp}\right)$; see Eqs. (B3) and (B7). The periodic boundary conditions $\hat{c}\left(j_{\|}\right)=$ $\hat{c}\left(N+j_{\|}\right)$are enforced by the quasimomentum quantization $\delta k_{n}=2 \pi n / N$.

We consider a waveguide attached to a single site along the edge in the longitudinal position $j_{\|} \equiv j_{\text {ex }}$. The coupling to the waveguide as described by standard input-output theory is entirely characterized by the decay rate $\kappa$. Since the edge state is the only state within the amplifier bandwidth, the coupling of the waveguide to a single site is equivalent to a direct coupling to the edge state at the same longitudinal position $j_{\mathrm{ex}}$ with the renormalized decay rate $\kappa^{\prime}=\kappa\left|u\left[j_{\perp}=1\right]\right|^{2}$. The resulting Langevin equation reads

$\dot{\hat{c}}\left(j_{\|}\right)=i\left[\hat{H}^{(\text {edge })}, \hat{c}\left(j_{\|}\right)\right]-\frac{\kappa^{\prime}}{2} \delta_{j_{\|}, j_{\mathrm{ex}}} \hat{c}\left(j_{\|}\right)+\sqrt{\kappa^{\prime}} \delta_{j_{\|}, j_{\mathrm{ex}}} \hat{a}^{(\mathrm{in})}$,

with the input-output relations

$$
\hat{a}^{\text {(out) }}=\hat{a}^{\text {(in) }}-\sqrt{\kappa^{\prime}} \hat{c}\left(j_{\mathrm{ex}}\right) .
$$

Next, we take the continuum limit by defining the chiral edge field

$$
\hat{c}(z)=\sqrt{\frac{1}{L}} \sum_{n=-\infty}^{\infty} e^{i \delta k_{n} j_{\|}} \hat{\alpha}_{\delta k},
$$

where $L$ is the edge length, and the periodic boundary conditions $\hat{c}(z)=\hat{c}(z+L)$ follow from the quasimomentum quantization $\delta k_{n}=2 \pi n / L$. From Eq. (B11) with $\tilde{E}=v \delta k, \Delta=0$, and $V(\delta k)=\nu$, and Eq. (D2), we find the field equations

$$
\begin{aligned}
\left(\partial_{t}+v \partial_{z}\right) \hat{c}(z)= & \nu \hat{c}^{\dagger}(z)-\frac{\kappa^{\prime}}{2} \delta\left(z-z_{\mathrm{ex}}\right) \hat{c}(z) \\
& +\sqrt{\kappa^{\prime}} \delta\left(z-z_{\mathrm{ex}}\right) \hat{a}^{(\mathrm{in})} .
\end{aligned}
$$

We note that, due to the point interaction with the waveguide, the field $\hat{c}(z)$ is not continuous at the position $z=z_{\mathrm{ex}}$ where the waveguide is attached, $\hat{c}\left(z_{\mathrm{ex}}+0_{+}\right) \neq$ $\hat{c}\left(z_{\mathrm{ex}}+0_{-}\right) \quad\left(0_{+}\right.$and $0_{-}$are infinitesimal positive and negative numbers, respectively). Keeping this in mind, the input-output relation in the continuous limit reads

$$
\hat{a}^{(\text {out })}=\hat{a}^{(\text {in })}-\sqrt{\kappa^{\prime}}\left[\hat{c}\left(0_{+}\right)+\hat{c}\left(0_{-}\right)\right] / 2 .
$$

\section{Impedance matching and gain}

Before investigating the interaction with the waveguide, we first discuss the propagation inside the ring. For concreteness, we consider $v>0$, such that $\hat{c}\left(z_{\mathrm{ex}}+0_{+}\right)$ $\left[\hat{c}\left(z_{\text {ex }}+0_{-}\right)\right]$is the field immediately after [before] the interaction with the waveguide. A signal travels an almost complete round-trip from $z=z_{\mathrm{ex}}+0_{+}$to $z=L+z_{\mathrm{ex}}+0_{+}=$ $z_{\mathrm{ex}}+0_{-}$in the time $t=L / v$. During this time no interaction with the waveguide takes place. From Eq. (D5), one readily finds

$$
\begin{aligned}
\hat{X}\left(z_{\text {ex }}+0_{-}, L / v+t_{0}\right) & =e^{\nu L / v} \hat{X}\left(z_{\text {ex }}+0_{+}, t_{0}\right), \\
\hat{Y}\left(z_{\text {ex }}+0_{-}, L / v+t_{0}\right) & =e^{-\nu L /|v|} \hat{Y}\left(z_{\text {ex }}+0_{+}, t_{0}\right) .
\end{aligned}
$$

Here, we introduce the amplified and deamplified quadratures, $\hat{X}=\left(\hat{c}^{\dagger}+\hat{c}\right) / \sqrt{2}$ and $\hat{Y}=i\left(\hat{c}^{\dagger}-\hat{c}\right) / \sqrt{2}$, respectively. We can conclude that a signal with the right phase during a complete round-trip inside the ring experiences a power gain

$$
G=e^{2 \nu L / v} .
$$

Next, we discuss the interaction with the waveguide. An input signal from the waveguide will be partly reflected and partly transmitted into the ring at $z=0_{+}$. Then, it will propagate inside the ring until it has completed a round-trip. A signal with the right phase will be amplified along the way. Before starting a new round-trip, part of the amplified signal returns into the waveguide. If the signal remaining in the ring at the beginning of the second round-trip is smaller compared to the signal in the ring at the beginning of the first round-trip, the signal will decay after few round-trips. In this regime, the waveguide stabilizes the edge state. By integrating the Heisenberg equations [Eq. (D5)] close to $z=0$, we find

$$
\left(\begin{array}{c}
\sqrt{|v|} \hat{c}\left(0_{+}\right) \\
\hat{a}^{\text {(out) }}
\end{array}\right)=\left(\begin{array}{cc}
r & t \\
-t & r
\end{array}\right)\left(\begin{array}{c}
\sqrt{|v|} \hat{c}\left(0_{-}\right) \\
\hat{a}^{(\text {in })}
\end{array}\right)
$$

where $r$ and $t$ are the reflection and transmission probability amplitudes:

$$
r=\frac{4-g^{2}}{4+g^{2}}, \quad t=\frac{4 g}{4+g^{2}}, \quad g=\sqrt{\frac{\kappa^{\prime}}{v}} .
$$

To prevent instability, we require that the transmission $t$ be large enough that the field in the edge-mode ring does not grow with each round-trip. The simplest case is where we tune the decay rate $\kappa$ so that all of the incident wave in the edge mode ends up in the coupling waveguide; i.e., $r=0, t=1$. This requires $g=2$, or in terms of the decay rate $\kappa$, the edge state velocity $v$, and the edge state transversal wave function at the edge $u\left(j_{\perp}=1\right)$, we find

$$
\kappa=\frac{4 v}{\left|u\left(j_{\perp}=1\right)\right|^{2}} \equiv \kappa_{\mathrm{id}} .
$$

If this impedance-matching condition is met, signals incident from the waveguide in the $\hat{X}$ quadrature will be reflected back with a power gain $G=G_{\max }$ independent of frequency. The impedance matching ensures that multiple traversals of the 
ring are impossible, which both precludes instability and also prevents the formation of standing wave resonances and a strongly frequency-dependent gain.

We note that the effective model we describe above can be straightforwardly extended to describe a chiral edge state coupled to several waveguides. In particular, we consider a setup with two impedance-matched waveguides at position $z=z_{\text {in }}$ and $z=z_{\text {out }}\left(z_{\text {out }}-z_{\text {in }}=L\right)$, respectively. A signal from the first waveguide entering the edge state at position $z=z_{\text {in }}$ is entirely transmitted into the second waveguide at position $z=z_{\text {out }}$. For the appropriate phase of the input signal, we have

$$
\hat{\mathcal{U}}^{\text {in }}(t)=\sqrt{v} \hat{X}\left(z_{\text {in }}+0_{+}, t\right),
$$

where $\hat{\mathcal{U}}^{\text {(in) }}$ is the input quadrature. We denote $\hat{\mathcal{X}}^{\text {out }}$, the corresponding amplified quadrature at $z=z_{\text {out }}$ :

$$
\hat{\mathcal{X}}^{\text {out }}(t)=-\sqrt{v} \hat{X}\left(z_{\text {out }}+0_{-}, t\right) .
$$

From Eq. (D5), one finds

$$
\left|\hat{\mathcal{X}}^{\text {out }}(t+L / v)\right|^{2}=G\left|\hat{\mathcal{U}}^{\text {out }}(t)\right|^{2},
$$

where the gain $G$ is given by Eq. (D7).

\section{Impedance mismatch}

If the coupling waveguides are not perfectly impedance matched as per Eq. (D10), signals incident on the waveguides from the edge state can be transmitted past the waveguide, and multiple traversals of the edge become possible. If such transmission becomes too strong, one can have instabilities. Using the effective 1D model with coupling to the three waveguides (as introduced above), we find that in the presence of impedance mismatch, the stability condition is

$$
e^{2 \nu L / v}\left(\frac{1-\frac{\kappa_{\text {in }}}{\kappa_{\text {id }}}}{1+\frac{\kappa_{\text {in }}}{\kappa_{\text {id }}}}\right)^{2}\left(\frac{1-\frac{\kappa_{\text {out }}}{\kappa_{\text {id }}}}{1+\frac{\kappa_{\text {out }}}{\kappa_{\text {id }}}}\right)^{2}\left(\frac{1-\frac{\kappa_{\text {ink }}}{\kappa_{\text {id }}}}{1+\frac{\kappa_{\text {sink }}}{\kappa_{\text {id }}}}\right)^{2}<1,
$$

where the ideal impedance-match value of the couplings is $\kappa_{\text {id }}$ [cf. Eq. (D10)]. This condition simply corresponds to requiring that there is no net gain for waves that successfully complete a round-trip along the edge. Considering the worst-case scenario, where all impedance mismatches are the same, and writing $\kappa_{\text {coupling }} / \kappa_{\mathrm{id}}=1+\epsilon$, the stability condition becomes

$$
\left(\frac{\epsilon}{2}+\epsilon\right)^{3}<\frac{1}{\sqrt{G}}
$$

where $G$ is the forward photon number gain of the amplifier defined in Eq. (D7). For a gain of roughly $20 \mathrm{~dB}$, we see that mismatches of $\approx \pm 15 \%$ do not lead to instabilities. Furthermore, for mismatches of $-10 \%$, impedance

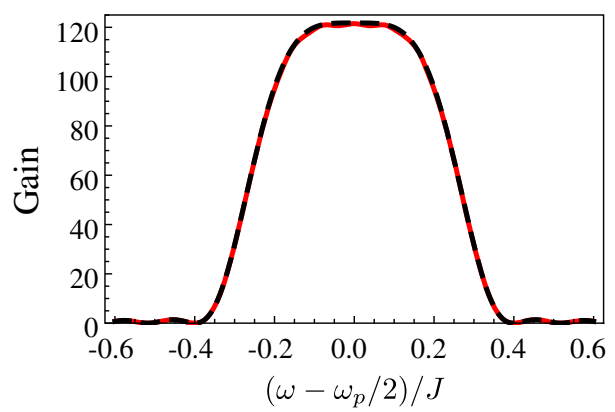

FIG. 6. Gain profile obtained in the effective 1D model with impedance-matched (black dashed line) and impedancemismatched (red solid line) waveguides. Small (almost invisible) ripples appear in the gain for the impedance-mismatched case. Parameters are total length 80 sites, length of the amplification region 30 sites, $\omega_{0}=2.14 \mathrm{~J}, \Phi=\pi / 2, \nu=0.08 \mathrm{~J}, \kappa=0.001 \mathrm{~J}$, impedance mistmatch $\epsilon=-0.1$ ).

mismatch induces small ripples (on the order of \pm 1 ) in the frequency-dependent gain (see Fig. 6). For even larger gains, one could stabilize the system engineering more sophisticated sinks. For example, one could have two or more impedance-matched sink waveguides. The gain after a round-trip will then decrease exponentially with the number of waveguides and will be reduced below unity, thus stabilizing the system in the presence of a sufficient number of waveguides. Thus, the lack of perfect impedance matching does not set any fundamental limit to the achievable gain.

\section{Added noise}

In order to show that our quantum amplifier design offers some degree of resilience to intrinsic losses and the corresponding noise, we calculate the added noise in the effective model. We consider a loss channel on each site and denote the corresponding decay rate as $\kappa_{\mathbf{j}}^{(\text {loss })}(z)$. In the continuous limit, and with the same approximations as above, we find the quantum field equations valid in the region between the input and output port:

$$
\begin{aligned}
& \left(\partial_{t}+v \partial_{z}\right) \hat{X}(z, t) \\
& =\left[\nu-\kappa^{(\text {loss })}(z) / 2\right] \hat{X}(z, t)+\sqrt{\kappa^{(\text {loss })}(z)} \hat{X}^{(\text {loss })}(z, t),
\end{aligned}
$$

where $\hat{X}^{(\text {loss })}(z, t)$ is the vacuum noise: $\left\langle\hat{X}^{(\text {loss })}(z, t)\right\rangle=0$ and

$$
\left\langle\hat{X}^{(\text {loss })}(z, t) \hat{X}^{(\text {loss })}\left(z^{\prime}, t^{\prime}\right)\right\rangle=\frac{1}{2} \delta\left(z-z^{\prime}\right) \delta\left(t-t^{\prime}\right) .
$$

We Fourier transform into frequency space and find the solution 


$$
\begin{aligned}
\hat{X}[z, \omega]= & e^{\left[(i \omega+\nu)\left(z-z_{\text {in }}\right)-\int_{z_{\text {in }}}^{z} \kappa^{(\text {loss })}\left(z^{\prime}\right) d z^{\prime} / 2\right] / v} \hat{X}\left(z_{\text {in }}+0_{+}, \omega\right) \\
& +\frac{1}{v} \int_{z_{\text {in }}}^{z} d z^{\prime} e^{\left[(i \omega+\nu)\left(z-z^{\prime}\right)-\int_{z^{\prime}}^{z} \text { (loss }^{(\text {los })}\left(z^{\prime \prime}\right) d z^{\prime \prime} / 2\right] / v} \\
& \times \sqrt{\kappa^{(\text {loss })}\left(z^{\prime}\right)} \hat{X}^{(\text {loss })}\left(z^{\prime}, \omega\right) .
\end{aligned}
$$

Putting together Eq. (D18) for $z=z_{\text {out }}-0_{-}$with Eqs. (D11) and (D12), we can relate the output signal $\hat{\mathcal{X}}^{\text {out }}$ to the input signal $\hat{\mathcal{U}}^{\text {in }}$ of the amplifier:

$$
\begin{aligned}
\hat{\mathcal{X}}^{\text {out }}[\omega]= & -e^{\left[(i \omega+\nu) L-\int_{z_{\text {in }}}^{z_{\text {out }}} \kappa^{(\text {loss })}\left(z^{\prime}\right) d z^{\prime} / 2\right] / v} \hat{\mathcal{U}}^{(\text {in })}[\omega] \\
& +\frac{1}{\sqrt{v}} \int_{z_{\text {in }}}^{z_{\text {out }}} d z^{\prime} e^{\left[(i \omega+\nu)\left(z_{\text {out }}-z^{\prime}\right)-\int_{z^{\prime}}^{z_{\text {out }}} \kappa^{(\text {loss })}\left(z^{\prime \prime}\right) d z^{\prime \prime} / 2\right] / v} \\
& \times \sqrt{\kappa^{(\text {loss })}\left(z^{\prime}\right)} \hat{X}^{(\text {loss })}\left(z^{\prime}, \omega\right) .
\end{aligned}
$$

The added noise $S_{\text {add }}(\omega)$ in noise quanta is, by definition,

$$
S_{\text {add }}(\omega)=\frac{S_{\mathcal{X}, X}(\omega)}{G(\omega)}-\frac{1}{2},
$$

where $S_{\mathcal{X}, X}(\omega)$ is the symmetrized noise at the output port,

$$
S_{\mathcal{X}, X}[\omega] \delta\left(\omega+\omega^{\prime}\right)=\frac{1}{2}\left\langle\left\{\hat{\mathcal{X}}[\omega], \hat{\mathcal{X}}\left[\omega^{\prime}\right]\right\}\right\rangle,
$$

and $G(\omega)$ is the gain. We note that in our simple approach $S_{\mathcal{X}, X}, G$, and $S_{\text {add }}$ are frequency independent. From Eqs. (D19) and (D17), we find

$$
G=e^{\left[2 \nu L-\int_{z_{\text {in }}}^{z_{\text {out }}} \kappa^{(\text {loss })}\left(z^{\prime}\right) d z^{\prime}\right] / v}
$$

and

$$
S_{\text {add }}=\frac{1}{v} \int_{0}^{L} d r e^{-\left[2 \nu r-\int_{0}^{L} \kappa^{(\text {loss })}\left(r+z_{\text {in }}\right) d r^{\prime}\right] / v} \kappa^{(\text {loss })}\left(r+z_{\text {in }}\right) .
$$

From this expression, we see that for $2 \nu>\kappa^{(\text {loss })}(z)$ the noise added at position $r+z_{\text {in }}$ is cut off exponentially as a function of the distance $r$ from the input. In particular, in the large gain limit and assuming that $\kappa^{(\text {loss })}(z)$ is smooth close to $z=z_{\text {in }}$, we can approximate

$$
S_{\text {add }} \approx \frac{\kappa^{(\text {loss })}\left(z_{\text {in }}\right)}{2 \nu} .
$$

Note, crucially, that as one increases the length $L$ of the amplifying channel, the gain increases exponentially, while the added noise remains constant. We thus see explicitly that the amplifying channel is immune to the majority of the internal loss noise in the system.

\section{Comparison between finite-size simulations and effective model}

In the regime where our effective theory applies (for signals well within the amplifier bandwidth), it can reproduce well the simulations of finite arrays. There are some quantitative differences due to well-understood finite-size effects. For instance, the edge state velocity is not constant in the finite-size array but rather decreases close to the edges where the edge state propagation changes direction. For this reason the gain does not depend only on the number of sites separating the input and the output port but also on the precise position of the ports. We place the input and output port close to the edges to enhance the gain. Indeed, the gain in the finite-size simulations is slightly larger than predicted by Eq. (D7). Because of the positiondependent edge state velocity, also the value of the decay rate required to obtain impedance matching depends on the position where the waveguide is attached (and is lower at the edges).

We note that the analytic continuum model can be extended to capture the frequency dependence of the gain and noise of our traveling wave amplifier; one needs, however, to incorporate into the model the leading-order quasimomentum dependence of the edge state velocity (which creates an effective pump detuning for the relevant parametric process). This will be presented in a future work.

[1] C. C. Gerry and P. L. Knight, Introductory Quantum Optics (Cambridge University Press, Cambridge, England, 2005).

[2] C. M. Caves, Quantum-Mechanical Noise in an Interferometer, Phys. Rev. D 23, 1693 (1981).

[3] B. Yurke, S. L. McCall, and J.R. Klauder, $S U(2)$ and $S U(1,1)$ Interferometers, Phys. Rev. A 33, 4033 (1986).

[4] V. Giovannetti, S. Lloyd, and L. Maccone, QuantumEnhanced Measurements: Beating the Standard Quantum Limit, Science 306, 1330 (2004).

[5] D. J. Thouless, M. Kohmoto, M. P. Nightingale, and M. den Nijs, Quantized Hall Conductance in a Two-Dimensional Periodic Potential, Phys. Rev. Lett. 49, 405 (1982).

[6] L. Lu, J. D. Joannopoulos, and M. Soljacic, Topological Photonics, Nat. Photonics 8, 821 (2014).

[7] Z. Wang, Y. Chong, J. D. Joannopoulos, and M. Soljacic, Observation of Unidirectional Backscattering-Immune Topological Electromagnetic States, Nature (London) 461, 772 (2009).

[8] T. Kitagawa, M. A. Broome, A. Fedrizzi, M. S. Rudner, E. Berg, I. Kassal, A. Aspuru-Guzik, E. Demler, and A. G. White, Observation of Topologically Protected Bound States in Photonic Quantum Walks, Nat. Commun. 3, 882 (2012).

[9] M. C. Rechtsman, J. M. Zeuner, Y. Plotnik, Y. Lumer, D. Podolsky, F. Dreisow, S. Nolte, M. Segev, and A. Szameit, Photonic Floquet Topological Insulators, Nature (London) 496, 196 (2013). 
[10] M. Hafezi, S. Mittal, J. Fan, A. Migdall, and J. M. Taylor, Imaging Topological Edge States in Silicon Photonics, Nat. Photonics 7, 1001 (2013).

[11] E. Prodan and C. Prodan, Topological Phonon Modes and Their Role in Dynamic Instability of Microtubules, Phys. Rev. Lett. 103, 248101 (2009).

[12] C. L. Kane and T. C. Lubensky, Topological Boundary Modes in Isostatic Lattices, Nat. Phys. 10, 39 (2013).

[13] V. Peano, C. Brendel, M. Schmidt, and F. Marquardt, Topological Phases of Sound and Light, Phys. Rev. X 5, 031011 (2015).

[14] Z. Yang, F. Gao, X. Shi, X. Lin, Z. Gao, Y. Chong, and B. Zhang, Topological Acoustics, Phys. Rev. Lett. 114, 114301 (2015).

[15] J. Paulose, B. G.-g. Chen, and V. Vitelli, Topological Modes Bound to Dislocations in Mechanical Metamaterials, Nat. Phys. 11, 153 (2015).

[16] R. Süsstrunk and S. D. Huber, Observation of Phononic Helical Edge States in a Mechanical Topological Insulator, Science 349, 47 (2015).

[17] L. M. Nash, D. Kleckner, A. Read, V. Vitelli, A. M. Turner, and W. T. M. Irvine, Topological Mechanics of Gyroscopic Metamaterials, Proc. Natl. Acad. Sci. U.S.A. 112, 14495 (2015).

[18] F. D. M. Haldane and S. Raghu, Possible Realization of Directional Optical Waveguides in Photonic Crystals with Broken Time-Reversal Symmetry, Phys. Rev. Lett. 100, 013904 (2008).

[19] S. Raghu and F. D. M. Haldane, Analogs of Quantum-HallEffect Edge States in Photonic Crystals, Phys. Rev. A 78, 033834 (2008).

[20] J. Koch, A. A. Houck, K. Le Hur, and S. M. Girvin, TimeReversal-Symmetry Breaking in Circuit-QED-Based Photon Lattices, Phys. Rev. A 82, 043811 (2010).

[21] R. O. Umucalılar and I. Carusotto, Artificial Gauge Field for Photons in Coupled Cavity Arrays, Phys. Rev. A 84, 043804 (2011).

[22] K. Fang, Z. Yu, and S. Fan, Realizing Effective Magnetic Field for Photons by Controlling the Phase of Dynamic Modulation, Nat. Photonics 6, 782 (2012).

[23] A. Petrescu, A. A. Houck, and K. Le Hur, Anomalous Hall Effects of Light and Chiral Edge Modes on the Kagomé Lattice, Phys. Rev. A 86, 053804 (2012).

[24] L. D. Tzuang, K. Fang, P. Nussenzveig, S. Fan, and M. Lipson, Non-Reciprocal Phase Shift Induced by an Effective Magnetic Flux for Light, Nat. Photonics 8, 701 (2014).

[25] M. Schmidt, S. Kessler, V. Peano, O. Painter, and F. Marquardt, Optomechanical Creation of Magnetic Fields for Photons on a Lattice, Optica 2, 635 (2015).

[26] M. Hafezi, E. A. Demler, M. D. Lukin, and J. M. Taylor, Robust Optical Delay Lines with Topological Protection, Nat. Phys. 7, 907 (2011).

[27] A. B. Khanikaev, S. Hossein Mousavi, W.-K. Tse, M. Kargarian, A. H. MacDonald, and G. Shvets, Photonic Topological Insulators, Nat. Mater. 12, 233 (2012).

[28] S. Mittal, J. Fan, S. Faez, A. Migdall, J. M. Taylor, and M. Hafezi, Topologically Robust Transport of Photons in a Synthetic Gauge Field, Phys. Rev. Lett. 113, 087403 (2014).
[29] R. Shindou, R. Matsumoto, S. Murakami, and J.-I. Ohe, Topological Chiral Magnonic Edge Mode in a Magnonic Crystal, Phys. Rev. B 87, 174427 (2013).

[30] G. Engelhardt and T. Brandes, Topological Bogoliubov Excitations in Inversion-Symmetric Systems of Interacting Bosons, Phys. Rev. A 91, 053621 (2015).

[31] C.-E. Bardyn, T. Karzig, G. Refael, and T. C. H. Liew, Chiral Bogoliubov Excitations in Nonlinear Bosonic Systems, Phys. Rev. B 93, 020502 (2016).

[32] V. Peano, M. Houde, C. Brendel, F. Marquardt, and A. A. Clerk, Topological Phase Transitions and Chiral Inelastic Transport Induced by the Squeezing of Light, Nat. Commun. 7, 10779 (2016).

[33] R. Barnett, Edge-State Instabilities of Bosons in a Topological Band, Phys. Rev. A 88, 063631 (2013).

[34] B. Galilo, D. K. K. Lee, and R. Barnett, Selective Population of Edge States in a 2D Topological Band System, Phys. Rev. Lett. 115, 245302 (2015).

[35] G. Engelhardt, M. Benito, G. Platero, and T. Brandes, Topological Instabilities in ac-Driven Bosonic Systems, Phys. Rev. Lett. 117, 045302 (2016).

[36] D. R. Hofstadter, Energy Levels and Wave Functions of Bloch Electrons in Rational and Irrational Magnetic Fields, Phys. Rev. B 14, 2239 (1976).

[37] M.Z. Hasan and C. L. Kane, Colloquium: Topological Insulators, Rev. Mod. Phys. 82, 3045 (2010).

[38] C. M. Caves, Quantum Limits on Noise in Linear Amplifiers, Phys. Rev. D 26, 1817 (1982).

[39] M. I. Dykman, M. Marthaler, and V. Peano, Quantum Heating of a Parametrically Modulated Oscillator: Spectral Signatures, Phys. Rev. A 83, 052115 (2011).

[40] V. Peano and M. Thorwart, Quasienergy Description of the Driven Jaynes-Cummings Model, Phys. Rev. B 82, 155129 (2010).

[41] M.-A. Lemonde and A. A. Clerk, Real Photons from Vacuum Fluctuations in Optomechanics: The Role of Polariton Interactions, Phys. Rev. A 91, 033836 (2015).

[42] M. Notomi, E. Kuramochi, and T. Tanabe, Large-Scale Arrays of Ultrahigh-Q Coupled Nanocavities, Nat. Photonics 2, 741 (2008).

[43] S. Mookherjea and A. Yariv, Coupled Resonator Optical Waveguides, IEEE J. Quantum Electron. 8, 448 (2002).

[44] B. J. Eggleton, B. Luther-Davies, and K. Richardson, Chalcogenide Photonics, Nat. Photonics 5, 141 (2011).

[45] J. Dahdah, M. Pilar-Bernal, N. Courjal, G. Ulliac, and F. Baida, Near-Field Observations of Light Confinement in a Two Dimensional Lithium Niobate Photonic Crystal Cavity, J. Appl. Phys. 110, 074318 (2011).

[46] A. H. Safavi-Naeini, S. Gröblacher, J. T. Hill, J. Chan, M. Aspelmeyer, and O. Painter, Squeezed Light from a Silicon Micromechanical Resonator, Nature (London) 500, 185 (2013).

[47] T. P. Purdy, P. L. Yu, R. W. Peterson, N. S. Kampel, and C. A. Regal, Strong Optomechanical Squeezing of Light, Phys. Rev. X 3, 031012 (2013).

[48] D. L. Underwood, W. E. Shanks, J. Koch, and A. A. Houck, Low-Disorder Microwave Cavity Lattices for Quantum Simulation with Photons, Phys. Rev. A 86, 023837 (2012). 
[49] B. M. Anderson, R. Ma, C. Owens, D. Schuster, and J. Simon, Engineering Topological Materials in Microwave Cavity Arrays, arXiv:1605.03177.

[50] T. Shi, H. J. Kimble, and J. I. Cirac, Topological Phenomena in Classical Optical Networks, arXiv:1603.03266v1.

[51] A. Metelmann and A. A. Clerk, Nonreciprocal Photon Transmission and Amplification via Reservoir Engineering, Phys. Rev. X 5, 021025 (2015).

[52] L. Ranzani and J. Aumentado, Graph-Based Analysis of Nonreciprocity in Coupled-Mode Systems, New J. Phys. 17, 023024 (2015).

[53] B. Abdo, K. Sliwa, S. Shankar, M. Hatridge, L. Frunzio, R. Schoelkopf, and M. Devoret, Josephson Directional Amplifier for Quantum Measurement of Superconducting Circuits, Phys. Rev. Lett. 112, 167701 (2014).
[54] K. M. Sliwa, M. Hatridge, A. Narla, S. Shankar, L. Frunzio, R. J. Schoelkopf, and M. Devoret, Reconfigurable Josephson Circulator/Directional Amplifier, Phys. Rev. X 5, 041020 (2015).

[55] C. Macklin, K. O’Brien, D. Hover, M. E. Schwartz, V. Bolkhovsky, X. Zhang, W. D. Oliver, and I. Siddiqi, A NearQuantum-Limited Josephson Traveling-Wave Parametric Amplifier, Science 350, 307 (2015).

[56] B. A. Bernevig and T. L. Hughes, Topological Insulators and Topological Superconductors (Princeton University Press, Princeton, NJ, 2013).

[57] A. A. Clerk, M. H. Devoret, S. M. Girvin, F. Marquardt, and R. J. Schoelkopf, Introduction to Quantum Noise, Measurement, and Amplification, Rev. Mod. Phys. 82, 1155 (2010). 